\title{
Suppression of a MEF2-KLF6 Survival Pathway by PKA Signaling Promotes Apoptosis in Embryonic Hippocampal Neurons
}

\author{
Jahan Salma ${ }^{1,2,4}$ and John C. McDermott ${ }^{1,2,3,4}$ \\ ${ }^{1}$ Department of Biology, ${ }^{2}$ Centre for Research in Mass Spectrometry, ${ }^{3}$ Muscle Health Research Centre, and ${ }^{4}$ Centre for Research in Biomolecular \\ Interactions, York University, York University, Toronto, Ontario M3J 1P3, Canada
}

In the mammalian nervous system, regulation of transcription factor activity is a crucial determinant of neuronal cell survival, differentiation, and death. The myocyte enhancer factor 2 (MEF2) transcription factors have been implicated in cellular processes underlying neuronal survival and differentiation. A core component of the MEF2 complex is the MEF2D subunit. Recently, we reported that cAMPdependent protein kinase (cAMP/PKA) signaling negatively regulates MEF2D function in myogenic cells. Here, we assessed whether cAMP signaling converges on the prosurvival role of MEF2D in Sprague Dawley rat embryonic (E18) hippocampal neurons. Initially, we observed that experimental induction of cAMP/PKA signaling promotes apoptosis in primary hippocampal neurons as indicated by TUNEL and FACS analysis. Luciferase reporter gene assays revealed that PKA potently represses MEF2D trans-activation properties in neurons. This effect was largely reversed by engineered neutralizing mutations of PKA phospho-acceptor sites on MEF2D (S121/190A). Krüppel-like factor 6 (KLF6) was identified as a key transcriptional target of MEF2 in hippocampal neurons, and siRNA-mediated knockdown of KLF6 expression promotes neuronal cell death and also antagonizes the prosurvival role of MEF2D. These observations have important implications for understanding the pathways controlling cell survival and death in the mammalian nervous system.

\section{Introduction}

During development of the CNS, a critical balance exists between the molecular pathways that control neuronal cell death and survival (Contestabile, 2002). Neuronal apoptosis fulfills a crucial role for normal organization of the brain through the elimination of excess neurons and synaptic connections (Contestabile, 2002; Kano and Hashimoto, 2009; Eroglu and Barres, 2010). Conversely, abnormal apoptosis contributes to progression of neurodegenerative diseases such as Alzheimer's and Parkinson's (Pfeiffer et al., 2010; Legradi et al., 2011; Chu et al., 2011). Thus, there is considerable interest in the functional characterization of prosurvival molecules in the CNS.

The transcription factor Myocyte Enhancer Factor 2 (MEF2) has been implicated as playing a pivotal role in neuronal survival as well as in the development, differentiation, and plasticity of the mammalian CNS (Leifer et al., 1994; Schulz et al., 1996; Heidenreich and Linseman, 2004; Pulipparacharuvil et al., 2008). The

Received July 14, 2011; revised Jan. 7, 2012; accepted Jan. 9, 2012.

Author contributions: J.S. and J.C.M. designed research; J.S. performed research; J.S. and J.C.M. analyzed data; J.S. and J.C.M. wrote the paper.

These studies were supported by grants from the Canadian Institutes of Health Research (CIHR) to J.C.M. We wish to thank Prof. Scott Friedman (Mount Sinai School of Medicine, New York, NY) and Dr. Nicolas. P. Koritschoner (Facultad de Bioquimica y Ciencias Biologicas, Universidad Nacional del Litoral, Santa Fe, Argentina) for providing KLF6 plasmids. We thank Dr. Sam Benchimol and his research group for assistance and use of equipment for FACS analysis.

The authors declare no competing financial interests.

Correspondence should be addressed to John C. McDermott, Department of Biology, 327 Farquharson, York University, 4700 Keele Street, Toronto, Ontario M3J 1P3, Canada. E-mail: jmcderm@yorku.ca.

DOI:10.1523/JNEUROSCI.3609-11.2012

Copyright $\odot 2012$ the authors $\quad 0270-6474 / 12 / 322790-14 \$ 15.00 / 0$
MEF2 transcription factor activity was originally identified in myogenic cells but has since been shown to function in multiple cell types including cardiac, skeletal, and smooth muscle, T-cells, and neurons (Ornatsky and McDermott, 1996; Black and Olson, 1998; Zhu et al., 2005; Potthoff and Olson, 2007). MEF2 functions as a transcriptional regulatory complex that integrates inputs from various signaling pathways (Yang et al., 1998; Naya and Olson, 1999; Cox et al., 2000; McKinsey et al., 2002). Expression of MEF2 in the brain is consistent with a role in neuronal differentiation and survival (Leifer et al., 1993; McDermott et al., 1993; Lyons et al., 1995; Lin et al., 1996). Disruption of molecular processes that control MEF2 transcriptional activity can indeed promote neuronal apoptosis (Li et al., 2001; Linseman et al., 2003; Butts et al., 2003; Bolger et al., 2007). The MEF2 family members (MEF2A, C, and D) are expressed throughout the developing and adult cerebellum and hippocampus (Ikeshima et al., 1995; Lin et al., 1996). Thus, signaling pathways that control MEF2 activity may exert control over neuronal cell survival pathways in the CNS. Recently, the MEF2 complex has also emerged as a key regulator of synapse development in the CNS (Shalizi et al., 2006; Flavell et al., 2006, 2008). RNA interference (RNAi) of MEF2A markedly decreases formation of synaptic structures in cerebellar granular neurons (Gaudilliere et al., 2002; Flavell et al., 2006).

The cAMP-dependent protein kinase A (PKA), an anciently conserved signaling molecule, regulates multiple biological processes (Belfield et al., 2006; Sands and Palmer, 2008). We reported an inhibitory effect of PKA on the trans-activation properties of MEF2D during myogenesis (Du et al., 2008). A role for PKA as a prosurvival kinase has been demonstrated in some cell types, 

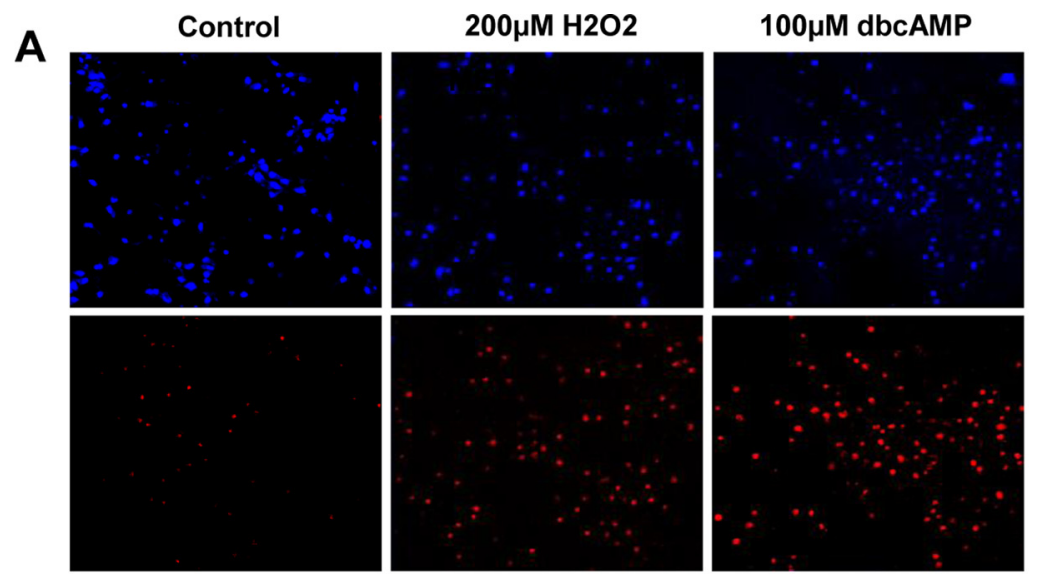

B
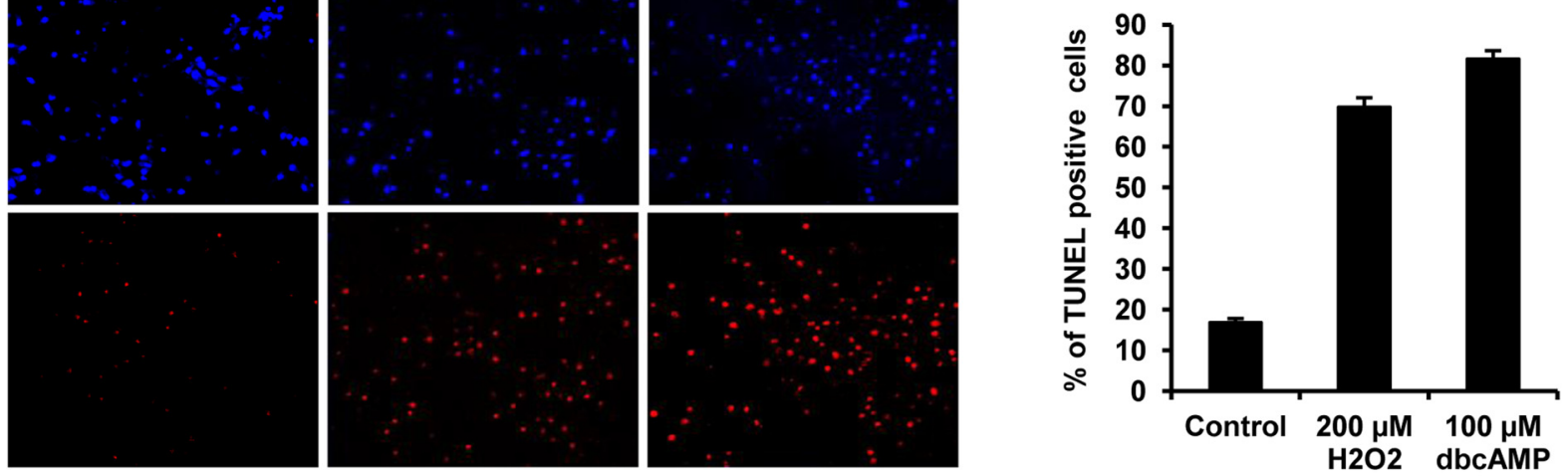

C

D
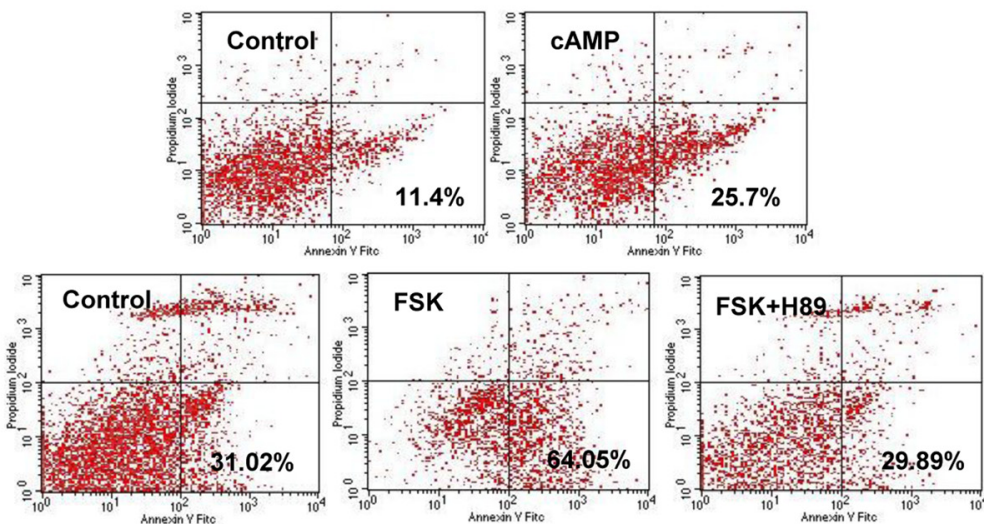

E

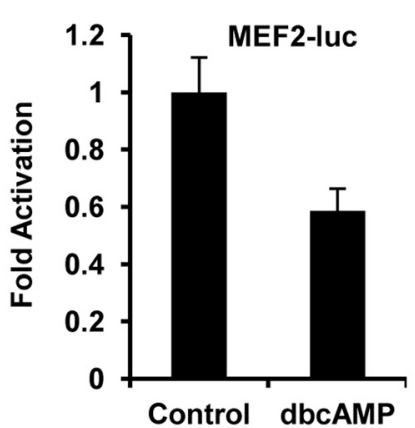

H

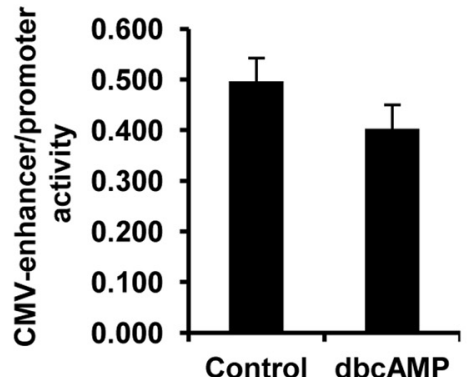

F

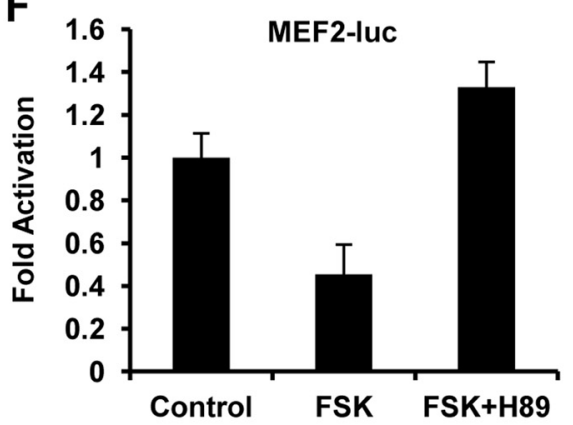

G 2.5
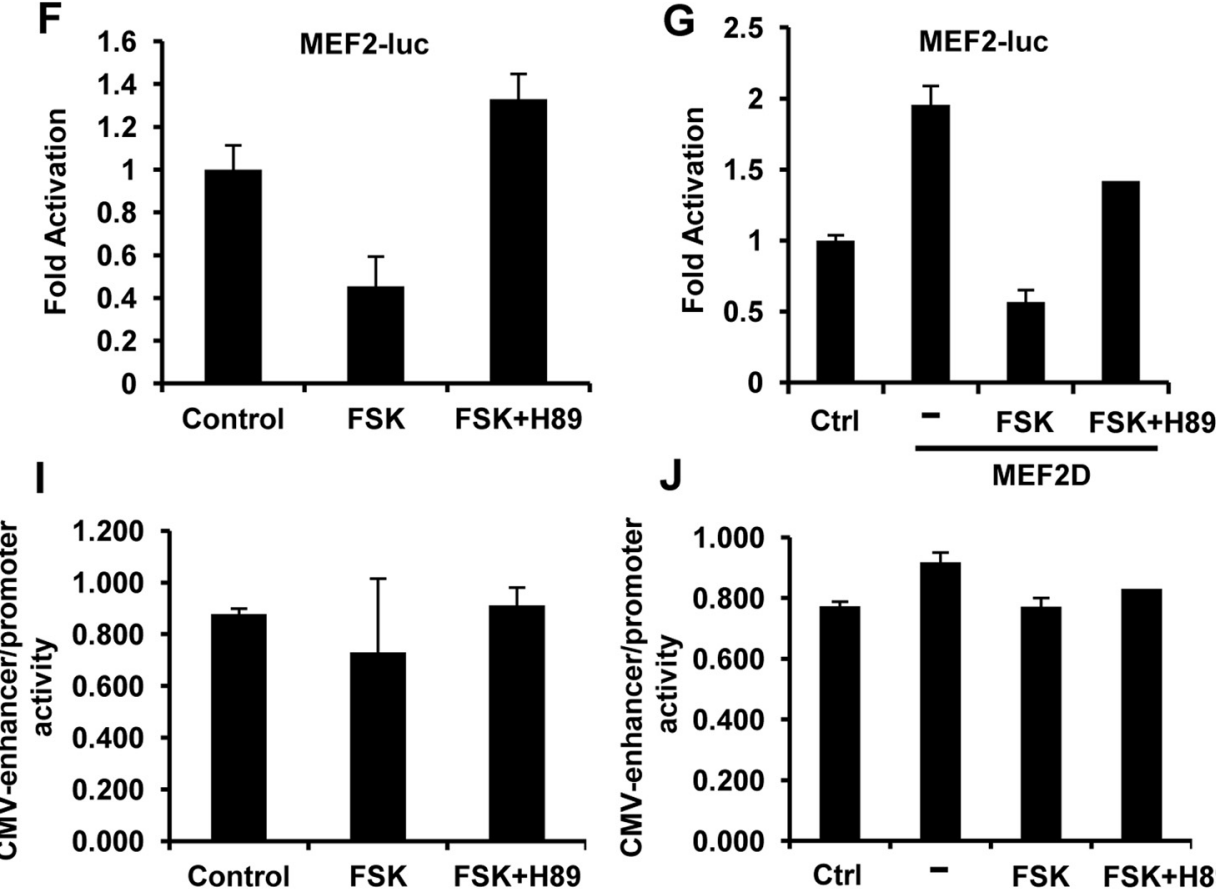

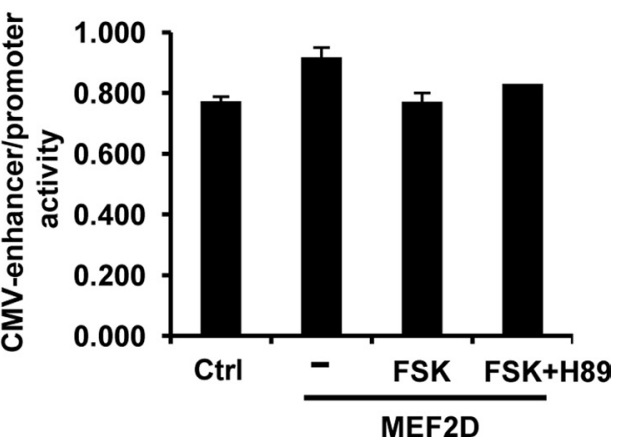

Figure 1. Activation of cAMP signaling induces apoptosis in hippocampal neurons. $A$, Primary hippocampal neurons (9DIV) were treated with a cAMP analog (100 $\mu$ M db-cAMPS, Sigma) for $6 \mathrm{~h}$ or $200 \mu \mathrm{M} \mathrm{H}_{2} \mathrm{O}_{2}$ (hydrogen peroxide) for $2 \mathrm{~h}$ as a positive control. Neuronal apoptosis was observed by using TUNEL assay. DNA fragmentation in apoptotic cells was visualized by terminal deoxyribonucleotidyl transferase-mediated dUTP-biotin nick end labeling. Representative images were taken from 10 randomly selected fields using fluorescence microscopy, both total (DAPI positive, a nuclear marker) and TUNEL-positive cells were counted. $\boldsymbol{B}$, Quantitation of these data represents the percentage of apoptotic cells in each condition. Data are expressed as mean \pm SEM from three separate treatments. C, Primary hippocampal neurons were stimulated with $100 \mu \mathrm{m} \mathrm{db}$-CAMP for $6 \mathrm{~h}$. Percentage of apoptotic cells was determined by PI and annexin V-FITC fluorescence staining using flow cytometry analysis (FACS analyzer). D, Primary hippocampal neurons were treated with $10 \mu \mathrm{m}$ FSK alone and in combination with $10 \mu \mathrm{m}$ H89 for $6 \mathrm{~h}$. Percentage of apoptotic cells were determined by PI and annexin V-FITC fluorescence staining using FACS analyzer. E, Primary E18 hippocampal neuronal cells were transiently transfected with pGL3-4XMEF2-Luc reporter gene and pCMV- $\beta$-galactosidase after $7 \mathrm{~d}$ in culture using the calcium phosphate precipitation method. Thirty-six hours after transfection, cells were treated with $100 \mu \mathrm{m}$ db-cAMP (Figure legend continues.) 
although it has also been implicated in hippocampal neuronal apoptosis (Zhao et al., 2008). Thus, we explored a connection between MEF2 and PKA in neuronal cells. Here, we report that repression of MEF2D by PKA signaling promotes neuronal apoptosis in hippocampal neurons. Also, we identify Krüppel-like factor 6 (KLF6) as a key downstream effector of the hippocampal MEF2 survival pathway.

\section{Materials and Methods}

Reagents and antibodies. Primary polyclonal antibodies $\alpha$-Actin (I-19), $\alpha$-KLF6 (R-173), and s.c.-7158 were purchased from Santa Cruz Biotechnology; $\alpha$ - $\beta$-tubulin III (Tuj 1) T3952, $\alpha$-HDAC4 (ML-19), H9411 were purchased from Sigma. Primary monoclonal antibodies $\alpha$ - $\beta$-tubulin III (T8660), and $\alpha$-MEF2D (610775), and GFP(B-2) (s.c.-9996) were purchased from Sigma, BD Biosciences, and Santa Cruz Biotechnology, respectively. Rabbit $\alpha$ - $\beta$-tubulin III (Tuj 1) T3952 was purchased from Sigma. Normal mouse (s.c.-2025), rabbit (s.c.-2027), and goat (s.c.2028) IgGs were purchased from Santa Cruz Biotechnology. FITC and TRITC-conjugated $\alpha$-rabbit and $\alpha$-mouse secondary antibodies were purchased from Sigma. Forskolin (F-3917), dbcAMP (D0260), H89dihydrochloride hydrate (B1427), Trichostatin A (T1952), DAPI (D9542), and $\mathrm{H}_{2} \mathrm{O}_{2}$ (H0904) were purchased from Sigma for use in cell culture. All other reagents were obtained as indicated herein.

Plasmids. Expression plasmids for full-length pcDNA3-MEF2D, pMT2 MEF2A, pMT2 MEF2C pCMV $\beta$-galactosidase, and MEF2 reporter gene constructs have been described in previous publications ( $\mathrm{Du}$ et al., 2008; Perry et al., 2009). The firefly luciferase reporter gene plasmid pGL3-4xMEF2-Luc was made with four copies of the MEF2 sites inserted. The expression vector of the catalytic subunit of PKA (pFC-PKA) and expression vector for FLAG-tagged HDAC4 were previously described (Du et al., 2008; Gordon et al., 2009; Perry et al., 2009). The HDAC4-eGFP and HDAC4 L175A plasmids were a kind gift from Dr. X.-J Yang (McGill University, Montreal, Quebec, Canada). Expression plasmids for pcDNA3-Flag-Zf9 and pCIneo-KLF6 were a kind gift from Prof. Scott Friedman (Mount Sinai School of Medicine, New York, NY). The Krüppel-like factor 6 (KLF6) reporter constructs pROM6, pROM5, pROM4, and pROM3-Luc were generously provided by Dr. Nicolas. P. Koritschoner (Facultad de Bioquimica y Ciencias Biologicas, Universidad Nacional del Litoral, Santa Fe, Argentina).

Embryonic hippocampal neuronal cell culture and transfection. Primary hippocampal neurons were isolated from timed-pregnant (E18) Sprague Dawley rat embryos (either sex) as described previously (Perry et al., 2009) in accordance with the Institutional Animal Care and Use Committee of York University. The hippocampi were collected in dissociation medium and digested with trypsin, followed by trituration. Neurons were plated on precoated poly-D-lysine (Sigma), six-well tissue culture plates. Neurons were maintained in Neurobasal Medium (Invitrogen) supplemented with B27 (2 ml/100 ml, Invitrogen) and L-glutamine (1 $\mathrm{ml} / 100 \mathrm{ml}$; Invitrogen), and penicillin $(50 \mathrm{U} / \mathrm{ml}) /$ streptomycin $(50 \mu \mathrm{g} /$ $\mathrm{ml}$ ) in a $37^{\circ} \mathrm{C}$ humidified incubator with a $5 \% \mathrm{CO}_{2}$ in air. After $24 \mathrm{~h}$, one-third of the culture medium was replaced with fresh medium and maintained for $7 \mathrm{~d}$ in vitro (7DIV) before transient transfection. In general, primary hippocampal neurons were transfected by using calcium phosphate-mediated transfection with indicated plasmids on day 6 or 7 in culture, and assays were conducted $36 \mathrm{~h}$ after transfection. Cells were

$\leftarrow$

(Figure legend continued.) or solvent (control) for 6 h. MEF2-mediated transcriptional activity was determined by Luciferase and $\beta$-galactosidase assay as described in Materials and Methods. Luciferase values were normalized by $\beta$-galactosidase activity. Experiments were conducted at least three times, yielding comparable results. $\boldsymbol{F}$, Primary hippocampal neuronal cells were transfected with pGL3- 4XMEF2-Luc reporter gene and PCMV- $\beta$-galactosidase. Cells were treated with $10 \mu \mathrm{m}$ FSK alone and in combination with $10 \mu \mathrm{m}$ H89 for $6 \mathrm{~h}$ (as indicated) (F, $\boldsymbol{G}$ ). Data indicating the activity of a generic enhancer/promoter are indicated in $\boldsymbol{H}$-J. Primary hippocampal neuronal cells were cotransfected with pGL3-4XMEF2-Luc reporter gene and wTMEF2D expression vector. MEF2-mediated transcriptional activation was determined by luciferase and $\beta$-gal assays.
A

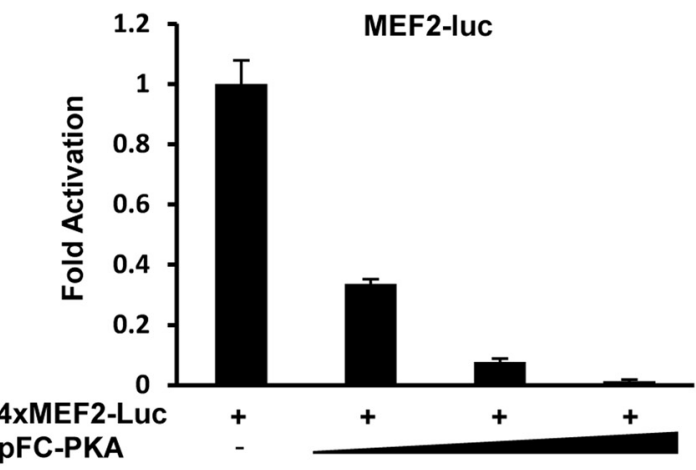

B

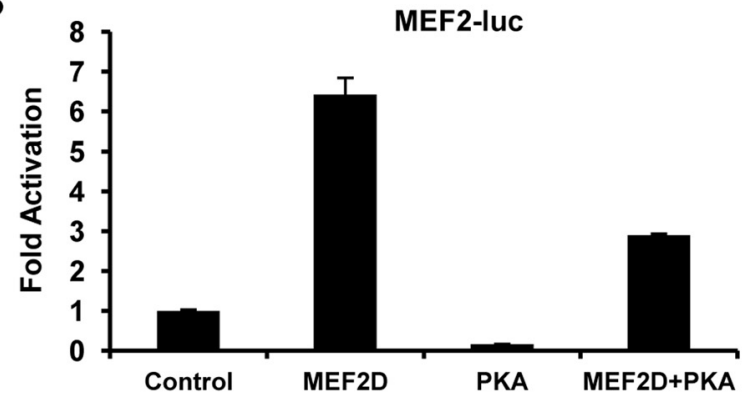

C

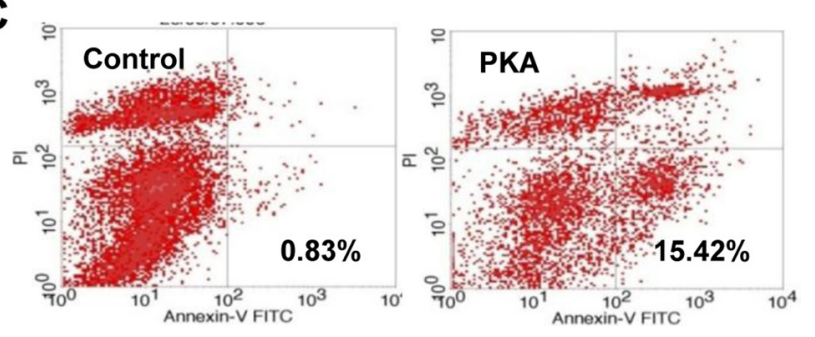

Figure 2. Exogenous expression of activated PKA suppresses the prosurvival role of MEF2 in hippocampal neurons. A, Primary E18 hippocampal neuronal cells (7DIV) were transiently transfected with pGL3-4XMEF2-Luc reporter gene, $\mathrm{pCMV}-\beta$-galactosidase to normalize transfection efficiencies, and increasing amounts of the catalytic subunit of PKA (pFC-PKA $250 \mathrm{ng}-1$ $\mu \mathrm{g}) . \boldsymbol{B}$, Primary hippocampal neuronal cells were cotransfected either with empty vector or pCDNA3-MEF2D, in combination with or without pFC-PKA (as indicated). MEF2-mediated transcriptional activity was determined by pGL3-4XMEF2-Luc reporter gene and PCMV- $\beta$ galactosidase assays. C, Primary hippocampal neuronal cells were cotransfected either with empty vector or pFC-PKA (as indicated). Thirty-six hours after transfection, primary hippocampal neuronal cells were stained with annexin V-FITC and PI (annexin V-FITC apoptosis detection kit, Sigma). Necrosis and apoptosis were determined by flow cytometry analysis. (The percentage of apoptotic cells labeled with annexin V-FITC appeared in the lower right quadrant of the density plot, shown in the bottom right corner of each panel).

replenished with fresh growth medium $24 \mathrm{~h}$ before transfection. The transfection efficiency was monitored by addition of a constitutively expressed GFP expression vector, typically yielding at least $50-60 \%$ transfected neuronal cells in each culture.

Cos7 cells were maintained in DMEM (Invitrogen ) with 10\% fetal bovine serum (FBS, HyClone), $1 \%$ penicillin-streptomycin, sodium pyruvate, and L-glutamine in a humidified incubator at $37^{\circ} \mathrm{C}$ and $5 \% \mathrm{CO}_{2}$ in air. Cells were seeded $1 \mathrm{~d}$ before transfection and transient transfections were performed using the standard calcium phosphate precipitation method. Cells were washed $16 \mathrm{~h}$ after transfection with PBS and harvested $48 \mathrm{~h}$ after transfection followed by preparation of lysate for reporter gene assays (see below).

TUNEL assay. TUNEL (terminal deoxynucleotidyl transferase UTPbiotin nick end labeling) staining was performed using In Situ Cell Death Detection Kit (Roche) according to the manufacturer's instructions. Primary hippocampal neurons were harvested and washed twice with PBS. Cells were trypsinized (0.2\%) and then fixed with 4\% PFA in PBS for $1 \mathrm{~h}$ at room temperature (RT). Cells were washed twice and permeabilized 
A

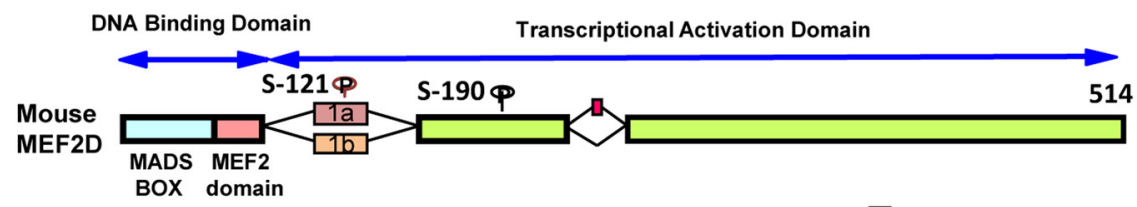

B
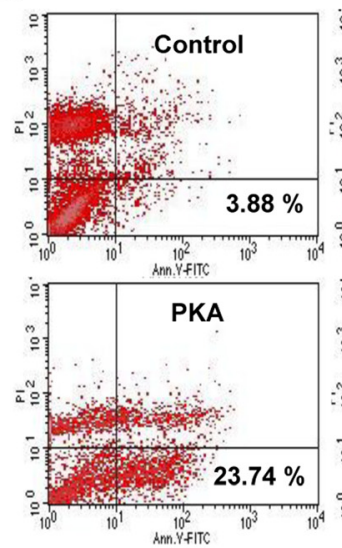

C

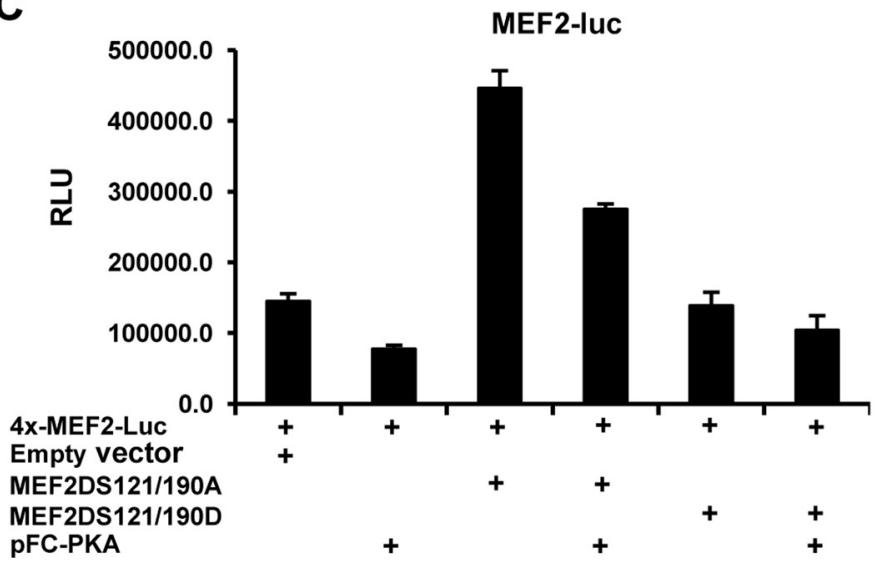

E
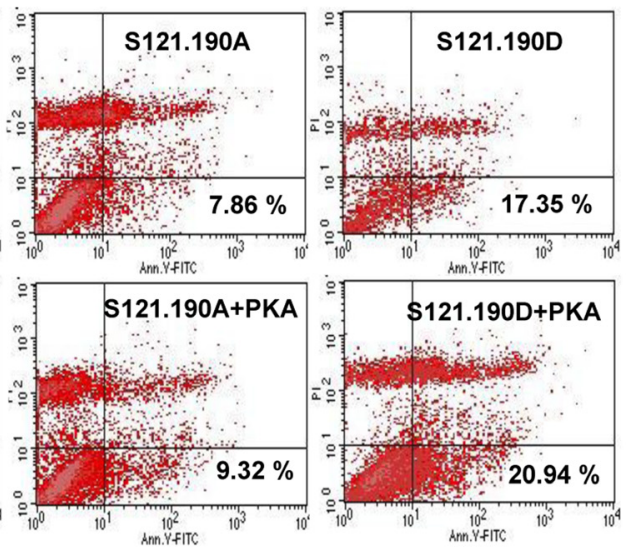

D

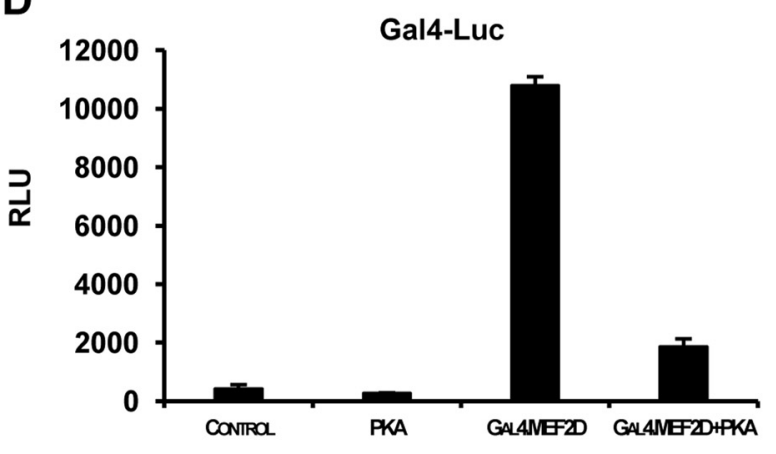

IB: MEF2D

IB: Actin

Figure 3. MEF2D-S121/190A confers resistance to PKA in hippocampal neurons. A, Schematic of mouse MEF2D indicating PKA phospho-acceptor sites. B, Primary hippocampal neuronal cells (7DIV) were transiently transfected with empty vector or mutated forms of MEF2D S121/190A (neutralizing) and S121/190D (phospho-mimetic) with or without PKA. Thirty-six hours after transfection, cells were stained with annexin V-FITC and PI (annexin V-FITC apoptosis detection kit, Sigma). Neuronal apoptosis was determined by flowcytometry analysis (FACS analyzer). C, Primary hippocampal neurons were transiently cotransfected at 7DIV with pGL3-4XMEF2-Luc reporter gene and pCMV- $\beta$-galactosidase to normalize transfection efficiencies and with empty vector or double mutated forms of MEF2D S121/190A and S121/190D, in combination with or without pFC-PKA (as indicated). MEF2-mediated transcriptional activity was determined by Luciferase and pCMV- $\beta$-Gal assays. D, Primary hippocampal neuronal cells were transiently cotransfected with 5XGAL4-Luciferase reporter vector, GAL-DBD or GAL4-MEF2D (87-507), with and without pFC-PKA and $\beta$-galactosidase to normalize transfection efficiencies. (Data are the mean $\pm \mathrm{SEM} ; n=3$ ). $\boldsymbol{E}$, Primary hippocampal neuronal cells (7DIV) were transiently transfected with empty vector or mutated forms of MEF2D S121/190A (neutralizing) and S121/190D with or without PKA. Cells were harvested and lysates were prepared $36 \mathrm{~h}$ later. Equal amounts of total protein were separated by 10\% SDS-PAGE followed by immunoblot analysis using MEF2D monoclonal antibody (1:1000) to detect the expression levels of transfected constructs. Actin (polyclonal, 1:2000) was used as a loading control.

with $0.1 \%$ Triton $\mathrm{X}-100$ for 4 min on ice followed by incubation with TUNEL assay reagent and propidium iodide (PI) for $1 \mathrm{~h}$ at $37^{\circ} \mathrm{C}$. Cells were washed with PBS twice and TUNEL-positive cells were quantified by fluorescence microscopy.

Reporter gene assays. Primary hippocampal neurons (7DIV) and COS7 cells were transiently transfected using the calcium phosphate precipitation method. Reporter gene plasmids $(1 \mu \mathrm{g})$ and expression plasmids $(1.5 \mu \mathrm{g})$ were transfected as indicated in Figures $1-11$. pCMV- $\beta$-galactosidase $(1 \mu \mathrm{g})$ was transfected as an internal control for monitoring transfection efficiency. The total amount of DNA for each experiment was kept constant by using empty vectors. Primary neuronal cells were seeded in 6-well plates for reporter gene assays. Neuronal cells were incubated with transfection reagent for $8 \mathrm{~h}$. Cells were harvested $36 \mathrm{~h}$ after transfection.

Cos7 cells were plated in 6-well plates $1 \mathrm{~d}$ before transfection. Transient transfection was performed using standard methods and cells were washed twice with PBS after $16 \mathrm{~h}$ transfection and then harvested at $48 \mathrm{~h}$ after transfection. Both $\beta$-galactosidase and luciferase activities were measured. Luciferase activity was assayed according to manufacturer's instructions (Promega), using a Berthold 9501 luminometer, and Luciferase values were normalized to $\beta$-galactosidase values. All measurements were made in triplicate for at least three independent experiments with data presented as means \pm SEM.

Western blot analysis. Protein extracts were prepared in NP-40 lysis buffer (0.5\% NP-40, $50 \mathrm{~mm}$ Tris-HCl [pH 8.0], $150 \mathrm{~mm} \mathrm{NaCl}, 1 \mathrm{~mm}$ EDTA [pH 8.0], $100 \mathrm{~mm}$ sodium fluoride, $10 \mathrm{~mm}$ sodium pyrophosphate) containing $0.2 \mathrm{~mm}$ PMSF and $0.5 \mathrm{~mm}$ sodium orthovanadate and protease inhibitor cocktail. Protein concentrations were determined by Bradford assay (Bio-Rad) with bovine serum albumin (BSA) as a standard. Equivalent amounts of total protein $(15-20 \mu \mathrm{g})$ were diluted in sample buffer (sodium dodecyle sulfate-polyacrylamide) containing $\beta$-mercaptoethanol, boiled for 4-5 min, and electrophoretically resolved by $10 \%$ SDS-PAGE gels, then electrophoretically transferred to an Immobilon-P membrane (Millipore). Nonspecific binding sites were blocked with 5\% milk in PBS for 1 hat RT. Immunoblotting was per- 
formed using appropriate primary antibodies in 5\% milk (PBS), $\alpha$-MEF2D (1:1000), $\alpha$-KLF6 (1:1000), $\alpha$-HDAC4 (1:1000), and $\alpha$-actin (1: 1000). The blots were then incubated with the appropriate secondary horseradish peroxidase (HRP) antibody (Bio-Rad) at 1:2000 with 5\% milk in PBS for $1 \mathrm{~h}$ at RT followed by chemiluminescence detection of immunoreactive proteins as per the manufacturer's instructions (GE Healthcare).

Coimmunoprecipitation assays. Protein extracts were prepared from primary hippocampal neurons as described above. Immunoprecipitation was performed using the ExactaCruz kit (Santa Cruz Biotechnology), as per manufacturer's instructions. Precipitated proteins were separated by SDS page and immunoblotting of precipitated proteins was performed as described above.

Flow cytometry analysis. Flow cytometry analyses were performed as previously described (Perry et al., 2009) using the annexin V-FITC apoptosis detection kit (Sigma) following the manufacturer's instructions. Cell viability and apoptosis were measured by a combination of annexin V-FITC and PI staining. Primary hippocampal neurons were washed and briefly trypsinized, and then washed twice with cold $1 \times$ PBS. Cells were pelleted by centrifugation and resuspended in $1 \times$ binding buffer followed by incubation with staining solution (annexin V-FITC and PI) for $15 \mathrm{~min}$ in the dark at $4^{\circ} \mathrm{C}$. The cells were resuspended in $1 \times$ binding buffer. Samples were kept on ice during the entire procedure and analyzed immediately by flow cytometry. Ten thousand cells from each sample were scanned and analyzed by FACS Calibur flowcytometry (Becton Dickinson) using the standard configuration and parameters. Data acquisition and analysis was performed using the CellQuest software (BD). Necrosis and apoptosis were determined by PI (FL2) and annexin V-FITC (FL1) fluorescence, respectively.

Site-directed mutagenesis. PCR-based mutagenesis of the MEF2 cis-element contained within the KLF6 promoter (pROM6) was performed by insertion of double-stranded oligonucleotides containing the mutated sequences, using the QuikChange site-Directed Mutagenesis kit (Stratagene, cat \# 200518) according to the manufacturer's instructions. Mutated constructs were verified by DNA sequencing (York University Core Facility).

Immunocytochemistry. Hippocampal neuronal cells were seeded on precoated poly-D-lysine glass coverslips at a density of $0.5 \times 10^{5}$ cells/ coverslip. After 7-9 d in vitro, cells were fixed in 4\% paraformaldehyde in PBS for $10 \mathrm{~min}$ at RT and then permeabilized with $0.3 \%$ Triton X-100 in PBS for 5 min. Cells were blocked with $10 \%$ goat serum in PBS for $30 \mathrm{~min}$ at $37^{\circ} \mathrm{C}$ and incubated overnight at $4^{\circ} \mathrm{C}$ with primary antibodies $\alpha$-MEF2D, $\alpha$-KLF6, $\alpha$-HDAC4, and $\alpha$ - $\beta$-tubulin III (1:100) diluted in $1.5 \%$ goat serum (PBS). Cells were washed $3 \times$ with PBS for $10 \mathrm{~min}$ and then incubated with the appropriate TRITC/FITC-conjugated secondary antibodies (1:500) in 1.5\% goat serum (PBS) for $2 \mathrm{~h}$ at RT following DAPI (4',6-diamidino-2-phenylindole) staining for $15 \mathrm{~min}$ at RT. Cells were washed $3 \times$ with PBS and coverslips were mounted with DAKO mounting media (Dako) on glass slides. The fluorescence images were captured using a Fluoview 300 (Olympus).

siRNA gene silencing. Small interfering RNAs (siRNAs) targeting KLF6, HDAC4, and a nonspecific scrambled RNA were purchased from (Sigma). Primary hippocampal neurons were seeded for FACS analysis and immunoblotting analysis. Cells were replenished with antibiotic-free Opti-MEM I (cat\# 31985, Invitrogen) media 2-3 h before transfection. Cells were trans-
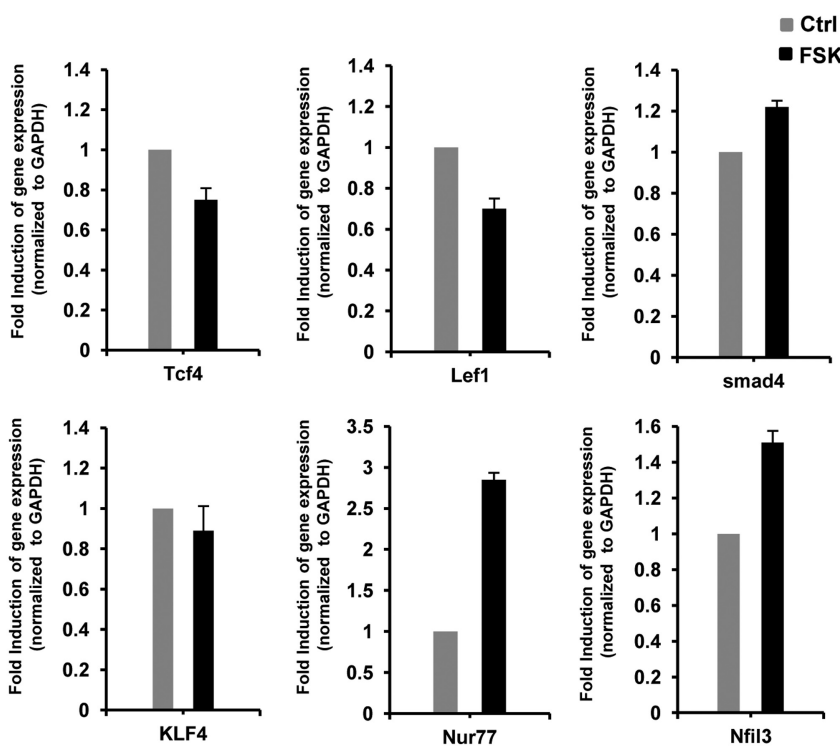

C

D

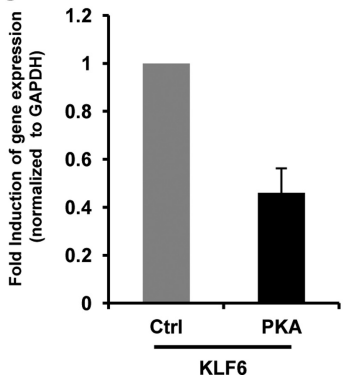

Hippocamp
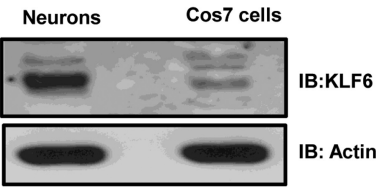

KLF6

Figure 4. KLF6gene expression in hippocampal neurons. $\boldsymbol{A}, \boldsymbol{B}$, Nine previously reported hippocampal MEF2 target genes were selected for qRT-PCRanalysis. Primary hippocampal neurons (7DIV) were stimulated with $10 \mu \mathrm{m}$ FSKor solvent for $6 \mathrm{~h}$ to induce PKA signaling. After or the callic subunit of PKA (pFC-PKA) followed by qRT-PCR analysis of KLF6 expression. D, KLF6 protein is expressed in hippocampal euronal cells. Cell lysates of primary hippocampal neurons and COS7 were prepared for immunoblotting analysis as indicated. Equal amounts of total protein were separated by 10\% SDS-PAGE followed by immunoblot. KLF6 polyclonal antibody (1:1000) was used to detect the endogenous KLF6 protein. Actin (polyclonal, 1:2000) was used as a loading control.

fected with siRNA and scrambled RNA using Lipofectamine RNAiMAX reagent (cat\# 13778, Invitrogen) according to the manufacturer's instructions. Cells were harvested $48 \mathrm{~h}$ after transfection for Western immunoblotting analysis to determine the efficacy of protein knock down or FACS analysis.

Quantitative real-time PCR. Total RNA was isolated from primary hippocampal neurons (7/9DIV) using the RNeasy kit (Qiagen) followed by DNase treatment (Qiagen) according to the manufacturer's protocol. All RNA samples were assessed for quality by agarose gel electrophoresis gels. Reverse transcription (RT) was performed from equal amounts of total RNA using Superscript III reverse transcriptase (Invitrogen) and used for semiquantitative RT-PCR analysis for primer specificity prior to quantitative real-time PCR (qRT-PCR) analysis. All quantitative RT-PCRs were performed on cDNA using Power SYBR Green Mastermix (Applied Biosystem) and detected using a 7500 fast real-time PCR system (Applied Biosytems) according to the manufacturer's protocol. All values were normalized to GAPDH (internal control) mRNA levels. Each experiment was done in triplicate and independently validated three times.

\section{Results}

cAMP signaling increases neuronal apoptosis

Previous studies have shown that activation of cAMP/PKA signaling in neuronal and non-neuronal cells can provoke apoptosis (Lømo et al., 1995; Myklebust et al., 1999; Zhao et al., 2008; 
A

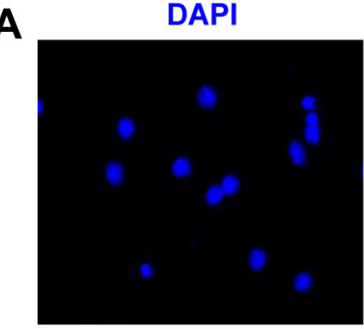

B

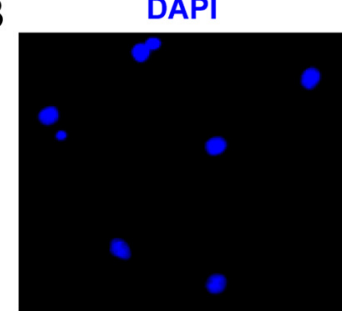

C

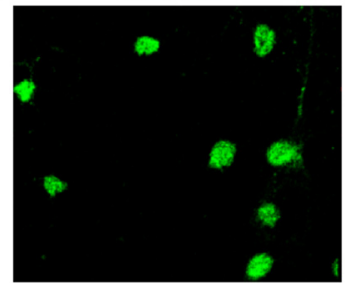

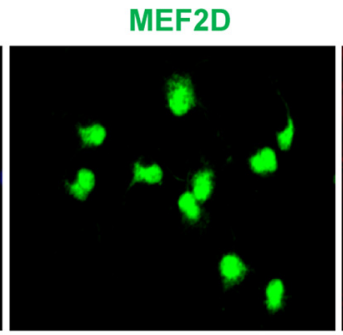

KLF6

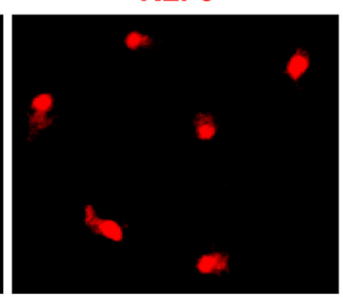

KLF6

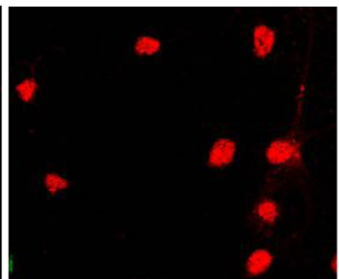

MEF2D / $\beta$-tubulin III

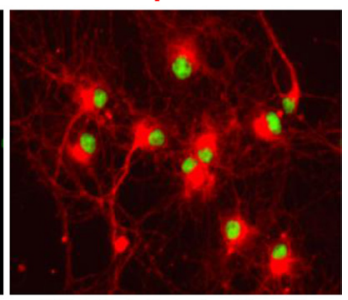

KLF6 / $\beta$-tubulin III

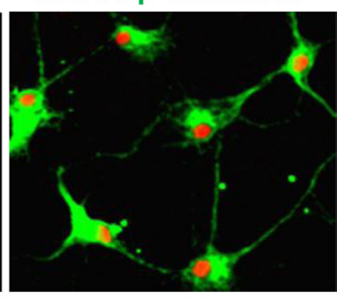

MEF2D / KLF6

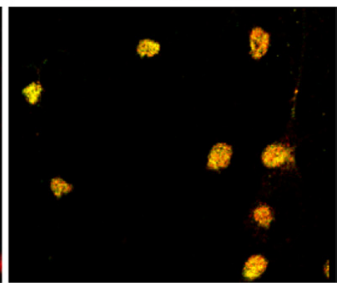

Figure 5. Cellular localization of MEF2D and KLF6 in hippocampal neurons. A, Primary hippocampal neuronal cells were fixed with $4 \%$ paraformaldehyde. Double immunofluorescence analysis was performed using primary antibodies to MEF2D (green) and a neuronal marker $\beta$ tubulin III (red). DAPI (4',6-diamidino-2-phenylindole) was used to identify nuclei (blue). The merged picture demonstrates localization of MEF2D (nuclear) and $\beta$-tubulin III positive cells (red). $\boldsymbol{B}$, Primary hippocampal neurons express KLF6. Hippocampal neuronal cells were fixed and double immunofluorescence analysis was performed with primary antibodies to KLF6 shown in red and to $\beta$-tubulin III shown in green. The merged picture demonstrates localization of KLF6 (nuclear) and $\beta$-tubulin III positive cells (green), counterstained with DAPI (blue). C, Double immunofluorescence labeling demonstrating KLF6 (red) and MEF2D (green) in primary hippocampal neurons. The merged picture indicates MEF2D-positive cells are mostly positive for KLF6 and both proteins are predominantly nuclear.

Zhang et al., 2008). MEF2D acts as prosurvival factor and we showed previously that it is expressed in the hippocampal neurons (Perry et al., 2009). In skeletal muscle, cAMP/PKA signaling has been shown to inhibit MEF2D function and myogenic differentiation (Du et al., 2008). We therefore undertook the current studies to determine whether the PKA-MEF2 pathway impinges on neuronal survival.

Initially, we performed a survival assay to examine the effect of cAMP-PKA signaling in primary hippocampal neurons (7DIV) using the TUNEL method. A cell-permeable cAMP analog was used to manipulate PKA activation (dbcAMP). Treatment of primary hippocampal neurons (9DIV) with dbcAMP indicated a prominent increase of TUNEL-positive cells when compared with untreated controls (Fig. $1 A$, bottom panels). Total cell numbers for each treatment are indicated by DAPI staining, a nuclear marker (Fig. 1A, top panels). Quantitative analysis shows that cAMP induced an approximately five- to six-fold increase in neuronal apoptosis compared with the control condition (Fig. $1 B$ ). Hydrogen peroxide $\left(\mathrm{H}_{2} \mathrm{O}_{2}\right)$ treatment was used in these assays as a positive control. Next, we assessed dbcAMP-mediated apoptosis in hippocampal neurons by FACS analysis. Estimation of necrosis and apoptosis were determined by a combination of PI and annexin V-FITC fluorescence, respectively (Fig. 1C). Cells appearing in the lower right quadrant of the density plots (high level of annexin $\mathrm{V}$-FITC and low level of PI), indicate an increase in the percentage of apoptotic cells in the cAMP-treated cells $(25.7 \%)$ compared with controls (11.4\%) (Fig. 1C). Forskolin treatment, which enhances

cAMP levels, also promotes apoptosis and a PKA inhibitor (H89) blocks this effect (Fig. 1D)

\section{PKA signaling represses MEF2 transcriptional activity in hippocampal neurons}

To obtain insight into whether the mechanism leading to apoptosis was through PKA inhibition of the prosurvival of MEF2D, we used a well known pharmacological inhibitor of PKA, H89, for $1 \mathrm{~h}$ before treatment with forskolin (FSK). We found that $\mathrm{H} 89$ blocked FSK effects and to some extent rescued neurons from apoptosis (Fig. 1D), and parallel reporter gene analysis demonstrated a reduction of MEF2 activity in FSK and dbcAMPtreated cells, which was reversed by $\mathrm{H} 89$ treatment (Fig. 1E-G). As an indicator of promoter specificity, we monitored the activity of a generic CMV enhancer/promoter reporter gene under the same experimental conditions (Fig. $1 H-J$ ). These data illustrate no demonstrable effect of PKA on this promoter construct, indicating that there is some specificity to the effects seen on the MEF2-dependent reporter gene. This specificity is also evident in the data shown later in Figure $4 A$ in which the message levels for a number of MEF2 target genes are unaffected by activation of PKA signaling, whereas some, such as KLF6, clearly are affected. Together, these data indicate that the effect of PKA activation is not a generalized downregulation of all transcribed genes. To further investigate the link between MEF2 transcriptional activity and PKA activation, primary hippocampal neurons (7DIV) were transiently transfected with a MEF2 reporter gene (MEF2-Luc) and increasing amounts of an expression plasmid encoding the catalytic subunit of PKA, which results in constitutive cellular PKA activity (pFC-PKA). It was observed that PKA potently suppressed MEF2-dependent reporter gene activation in a dose-dependent manner (Fig. 2A). Also, exogenous expression of wild-type (wt) MEF2D increased MEF2 reporter gene activation and PKA repressed this activation (Fig. 2B). To further investigate whether PKA plays a direct role in neuronal survival/apoptosis, neurons were transiently transfected with empty vector or pFC-PKA followed by FACS analysis. Substantial increases in apoptotic cells were observed with PKA (15.42\%) (Fig. 2C, right panel) when compared with control (0.83\%) (Fig. 2C). In congruence with the results seen with dbcAMP and activated PKA, similar results were also seen with FSK treatment, which activates adenylate cyclase (data not shown). Together, these results demonstrate that cAMP/PKA signaling regulates MEF2 activity and also potentiates apoptosis in hippocampal neurons.

\section{The role of MEF2 in hippocampal neuronal survival}

Our observations at this point suggested that PKA signaling might target the prosurvival role of MEF2. Previously, we have documented that PKA directly phosphorylates S121 and S190 on 
MEF2D using mass spectrometry (Fig. 3A), and these sites were sufficient to confer repressive effects on the skeletal muscle differentiation program in response to cAMP signaling (Du et al., 2008). Moreover, neutralization of these phospho-acceptor sites by mutation to Alanine (A) rendered MEF2D resistant to PKA signaling and thus allowed rescue of differentiation when the PKA resistant MEF2D was transfected into cells even in the presence of cAMP signaling (Du et al., 2008). We therefore reasoned that this PKA resistant form of MEF2D might be capable of rescuing hippocampal neuronal cells from cAMP-mediated apoptosis if the primary target of cAMP-PKA signaling is indeed MEF2D. Primary hippocampal neurons were transfected with empty vector or mutated forms of MEF2D S121/190A (neutralizing) and S121/190D (phospho-mimetic) with or without PKA at 7DIV (see Fig. $3 \mathrm{~A}$ for schematic of where these phosphoacceptor sites are in relation to other domains). Subsequently, FACS analysis was performed using annexin-V/PI to assess cell death. Interestingly, a decrease in apoptotic cells was observed when S121/190A was coexpressed with PKA compared with PKA alone in hippocampal neurons (Fig. 3B). Conversely, phosphomimetic forms of MEF2D, S121/190D failed to alter PKAmediated cell death and, if anything, showed an increase in the percentage of apoptotic cells even in the absence of PKA when compared with the control condition (Fig. $3 B$ ). These data support the idea that a PKA-resistant MEF2D (S121/190A) protects neurons from PKA-mediated cell death. This effect is corroborated by reporter gene analysis in which a Gal4-MEF2D fusion protein is repressed by PKA, and also that MEF2D S121/190A neutralizing mutation is much more resistant to the effects of PKA than wild-type MEF2 (Fig. 3C,D). Expression levels of the various mutated and wild-type MEF2D protein are shown in Figure $3 E$.

\section{Characterization of MEF2 target genes in hippocampal neuronal cells}

We next assessed the prosurvival effect of MEF2D and its inhibition by cAMP/PKA signaling by analyzing downstream MEF2 target genes identified in a study in which a high throughput chromatin immunoprecipitation method coupled to massively parallel sequencing (ChIP-seq) was used (Flavell et al., 2008). To directly examine whether the expression of KLF6 is targeted under conditions when MEF2 activity is repressed by PKA signaling, primary hippocampal neurons (7DIV) were treated with FSK or solvent for $6 \mathrm{~h}$ to induce PKA signaling. After stimulation, total RNA was isolated using RNeasy kit (Qiagen) followed by qRTPCR using Power SYBR Green Master mix. We performed qRTPCR analysis of a number of these identified MEF2 target genes to identify which ones might be affected by PKA activation (Fig. $4 A, B)$ and, from this analysis, a prominent responder was KLF6. Other reported MEF2 target genes showed variable responses to FSK treatment suggesting that MEF2 might play a different role at these genes (Fig. 4A,B). Primary hippocampal neurons (7DIV) were also transfected with empty vector or the catalytic subunit of PKA (pFC-PKA) followed by qRT-PCR analysis. Again, this showed that KLF6 mRNA level was suppressed by activated PKA (Fig. 4C). These results suggested that the decrease in KLF6 gene expression by PKA signaling might be through MEF2 inhibition. KLF6 is highly expressed in various regions of the brain including the hippocampus (Fischer et al., 2001; Jeong et al., 2009). We therefore sought to define whether KLF6 might constitute an important downstream effector of the MEF2 prosurvival role in hippocampal neurons. Endogenous expression of KLF6 is detected in E18 hippocampal neuronal cells (Fig. 4D), and immu-
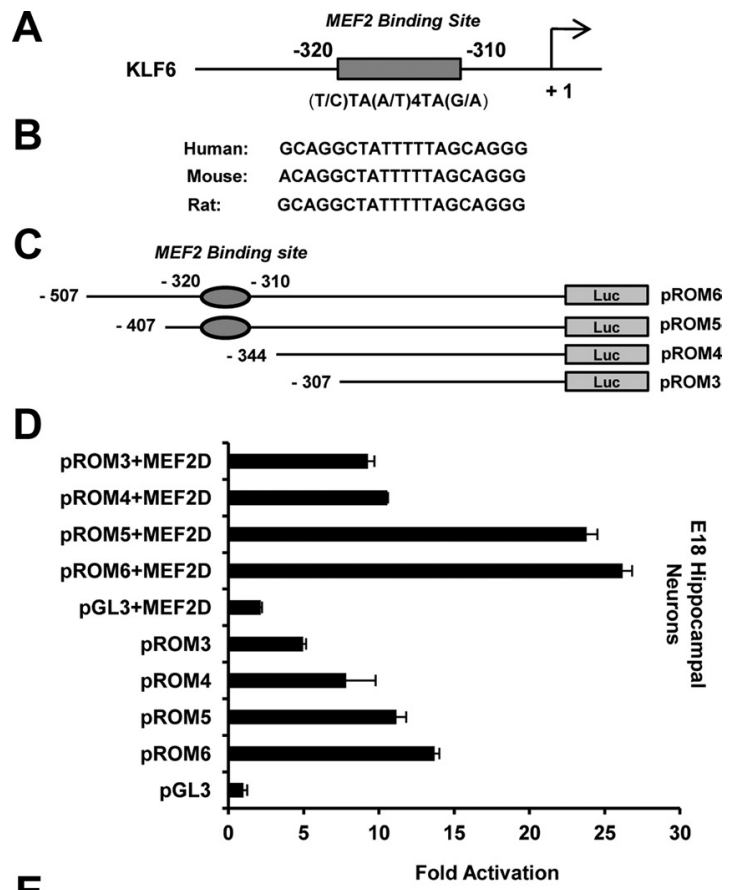

$\mathbf{E}$

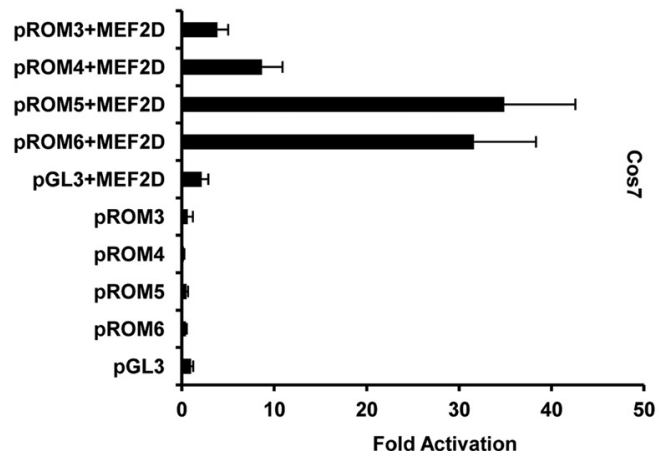

Figure 6. Functional analysis of KLF6 promoter in hippocampal neurons. A, Schematic illustration of upstream KLF6 promoter construct depicts location of MEF2 cis-element between -320 and -310 bp. $\boldsymbol{B}$, Sequence of MEF2 cis-element is highly conserved in the KLF6 promoter from human to mouse. C, Schematic illustrations of KLF6 reporter deletion constructs used in reporter assays. All KLF6 promoter constructs were cloned into the pGL3-basic reporter vector (pGL3-KLF6-Luc) and were used in examining KLF6 promoter activity. D, Primary hippocampal neuronal cells were transiently cotransfected with various constructs of the KLF6 promoter (pROM6 to pROM3); pCMV- $\beta$-galactosidase was used to normalize transfection efficiencies. Cell extracts were prepared and analyzed for luciferase and $\beta$-gal assays. $\boldsymbol{E}$, Cos7 cells were transiently cotransfected with various KLF6 promoter constructs (pROM6 to pROM3) and $\mathrm{pCMV}$ - $\beta$-galactosidase (to normalize transfection efficiencies) with and without wtMEF2D by using calcium phosphate method. pGL3-basic empty vector was used as a control. Cell extracts were prepared for luciferase and $\beta$-gal assays as described in Material and Methods. (Data are the mean \pm SEM; $n=3$.)

nofluorescence labeling to observe the cellular localization of KLF6 and MEF2D in primary hippocampal neurons was also carried out. Primary hippocampal neurons, confirmed by $\beta$-tubulin III expression (red), were positive for nuclear MEF2D (green) (Fig. 5A). Neuronal cells also show strong nuclear localization of KLF6 (red) in $\beta$-tubulin III positive cells (green) (Fig. $5 B$ ). Nuclear colocalization of MEF2D and KLF6 in primary hippocampal neurons is evident (Fig. 5C). Interestingly, the colocalization of MEF2D and KLF6 that we have observed in primary cultured neurons is also reflected in data from the Allen Brain Atlas Resources in which in situ hybridization of KLF6 and MEF2D show strikingly similar patterns of expression in the hippocampal region (available at http://www.brain-map.org). 
A

B
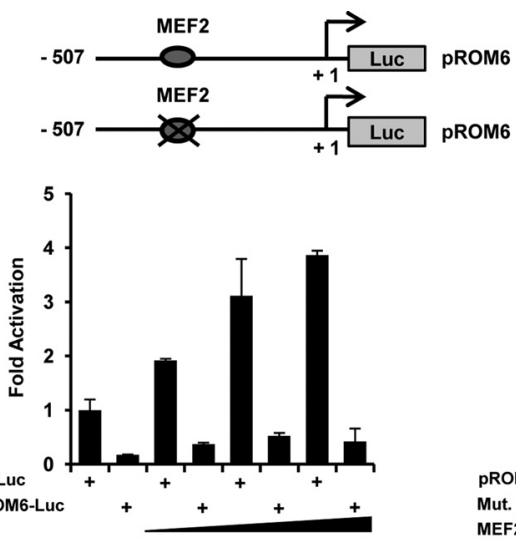

D

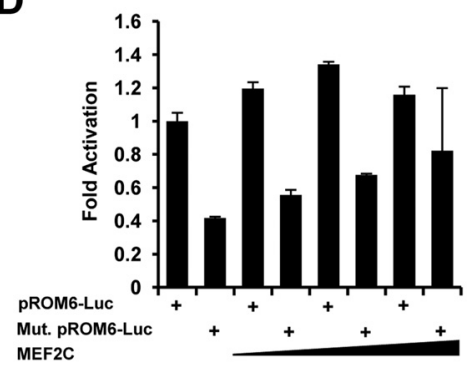

C

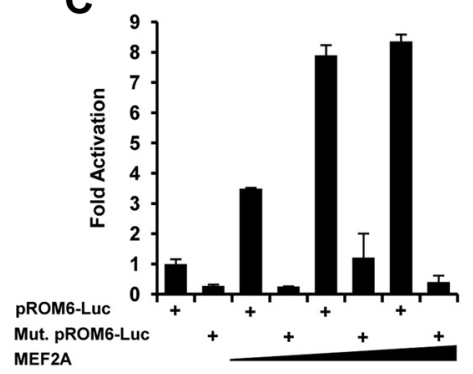

$E$

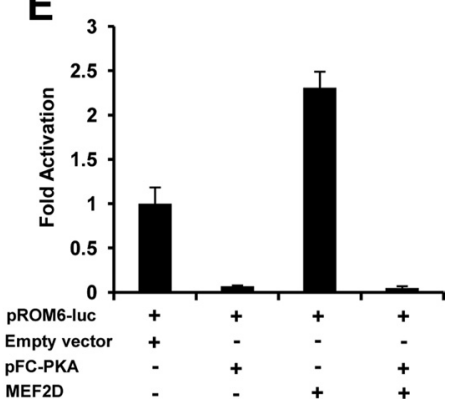

Figure 7. PKA represses KL6 promoter activity in primary hippocampal neurons. $\boldsymbol{A}$, Schematic illustrations of KLF6 promoter construct (pROM6) with intact MEF2-binding site or with MEF2 site mutated. $\boldsymbol{B}$ - $\boldsymbol{D}$, Primary hippocampal neurons were cotransfected with PROM6 reporter constructs and increasing amounts (250 ng to $1.5 \mu \mathrm{g}$ ) of wTMEF2D, MEF2A, and MEF2C plasmid, respectively. pCMV- $\beta$-galactosidase was used to normalize transfection efficiencies. $E$, Primary hippocampal neurons were transiently transfected with pGL3-KLF6-Luc reporter construct, pCMV- $\beta$-galactosidase, and wTMEF2D with or without pFC-PKA (as indicated). MEF2-mediated transcriptional activity was determined by luciferase and $\beta$-gal assays as described in Material and Methods. (Data are the mean \pm SEM; $n=3$.)

\section{MEF2D regulates KLF6 promoter activity in} hippocampal neurons

To further define the potential role of MEF2D in KLF6 gene regulation, we examined the previously characterized upstream KLF6 promoter (Gehrau et al., 2005) that contains a MEF2 ciselement, between -320 and -310 bp (Fig. $6 A$ ). This site is highly conserved in different species as depicted in Figure $6 \mathrm{~B}$. The conservation of this cis-element suggests that MEF2 may play an evolutionary conserved role in KLF6 gene expression. To investigate MEF2D-dependent regulation of the KLF6 promoter in hippocampal neurons, we used a number of KLF6 reporter gene constructs containing different fragments of the KLF6 promoter, pROM6 ( $-507 \mathrm{bp}$ to $+1 \mathrm{bp})$, pROM5 ( $-407 \mathrm{bp}$ to $+1 \mathrm{bp})$, and pROM4 ( -344 bp to $+1 \mathrm{bp})$, which contain the MEF2 ciselement, and pROM3 ( $-307 \mathrm{bp}$ to $+1 \mathrm{bp}$ ), which does not (schematic illustrations of KLF6 reporter deletion constructs are shown in Fig. 6C). All KLF6 fragments were cloned into the pGL3-basic reporter vector (pGL3-KLF6-Luc). Reporter gene assays were undertaken to assess MEF2D transactivation of the KLF6 promoter. Primary hippocampal neurons were transiently transfected with the KLF6 promoter constructs (pROM6 to pROM3) with and without wtMEF2D, and pGL3-basic empty vector was used as a control. As shown in Figure $6 D$, ectopically expressed MEF2D strongly induced pROM6 and pROM5 reporter transcriptional activity. MEF2D did not potentiate KLF6pROM3 reporter activity, which lacks the MEF2-binding site (Fig. 6D,E).

We wanted to precisely dissect the absolute requirement for the MEF2 cis-element in the KLF6 promoter and therefore per- formed site-specific mutation of the pROM6 MEF2 site. Primary hippocampal neurons were cotransfected with MEF2D and the pROM6 reporter construct containing the intact or mutated MEF2binding site as shown in Figure $7 A$. MEF2D did not induce KLF6 reporter activity when the MEF2-binding site was mutated to a sequence that no longer fits the consensus binding site (Fig. 7B). Furthermore, increasing amounts of MEF2D induced a dose-dependent increase in pROM6 reporter activity compared with the mut.pROM6-luc, which was not activated by MEF2D (Fig. 7B). To further determine whether other MEF2 isoforms (MEF2A and C) can alter KLF6 promoter activity, we performed reporter gene assays as described above using pROM6 reporter gene constructs containing the intact or mutated MEF2-binding site specific mutation. Increasing amounts of MEF2A induce pROM6 reporter gene activity compared with the mut.pROM6-luc reporter, in which the MEF2 site is inactivated by mutation (Fig. 7C). Conversely, although $\mathrm{MEF} 2 \mathrm{C}$ is a reasonably strong transactivator on synthetic and other natural promoters, it had essentially no effect on pROM6 (Fig. 7D). There are differences between MEF2A, C, and D in their ability to heterodimerize with each other, warranting further analysis of these observations. We next assessed whether PKA signaling targets the KLF6 promoter through the MEF2 cis- element. To investigate this, hippocampal neuronal cells were cotransfected with wtMEF2D and pROM6 $(-507 \mathrm{bp}$ to $+1 \mathrm{bp})$ in the presence and absence of pFC-PKA. The activity of these reporter genes was markedly reduced by PKA. The wild-type MEF2D was not able to reverse the inhibitory effect of PKA when ectopically expressed, which is consistent with our previous studies showing that PKA repression transdominantly represses exogenously expressed MEF2D (Fig. 7E). Together, these data indicate that MEF2D, possibly in combination with MEF2A, is a potent transcriptional regulator of the KLF6 promoter and this promoter can be repressed by PKA signaling through its MEF2 cis-element.

Interestingly, we observed that exogenous overexpression of KLF6 can reduce the amount of cell death provoked by PKA, suggesting it might play an important survival role downstream of MEF2 in hippocampal neurons (Fig. 8A, B). To further investigate MEF2-mediated regulation of KLF6 by PKA, we performed reporter gene assays using pGL3-KLF6-Luc (pROM6) and double mutations of MEF2D (S121.190A or S121.190D). Cos7 cells were cotransfected with wtMEF2D and MEF2D double mutants (S121.190A or S121.190D) with or without pFCPKA. Consistent with our previous observations, the MEF2D double mutation S121.190A is partially resistant to PKA (Fig. $8 C$ ). However, as observed before (Du et al., 2008), phosphomimetic mutations are still partially responsive to PKA, suggesting an indirect mechanisms may be involved in MEF2mediated KLF6 gene regulation. 
A
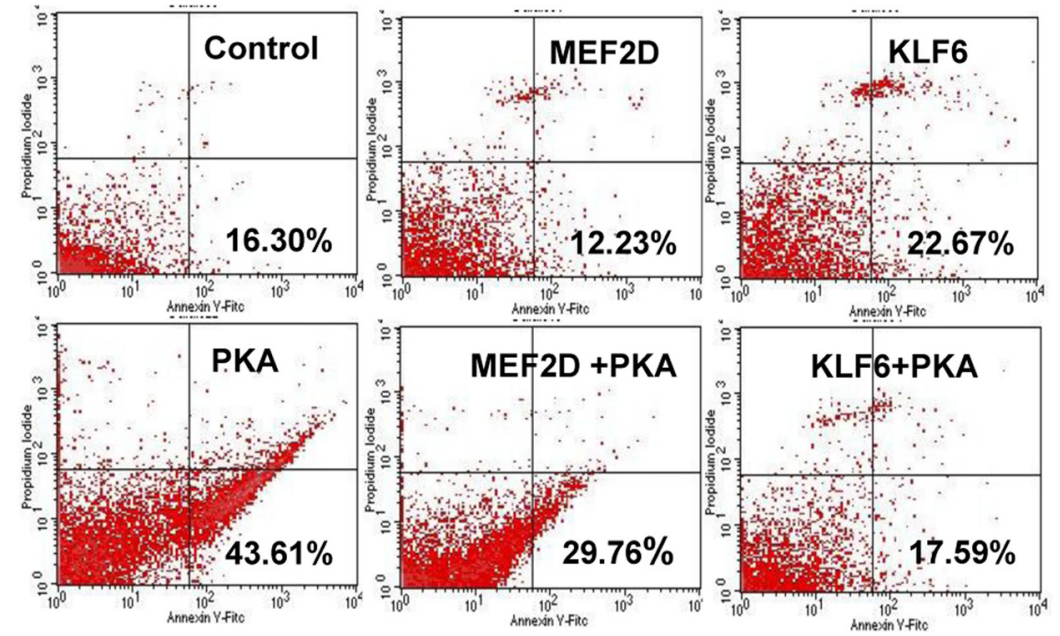

B

\section{Hippocampal neurons (9DIV)}

pcDNA3

KLF6

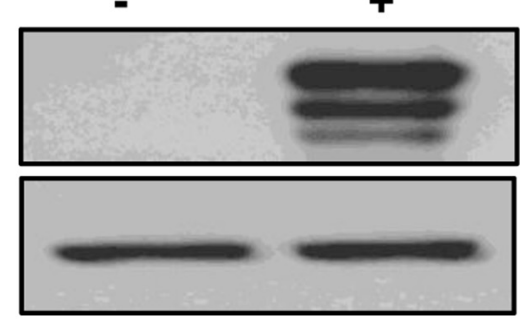

IB:KLF6

IB: Actin

C
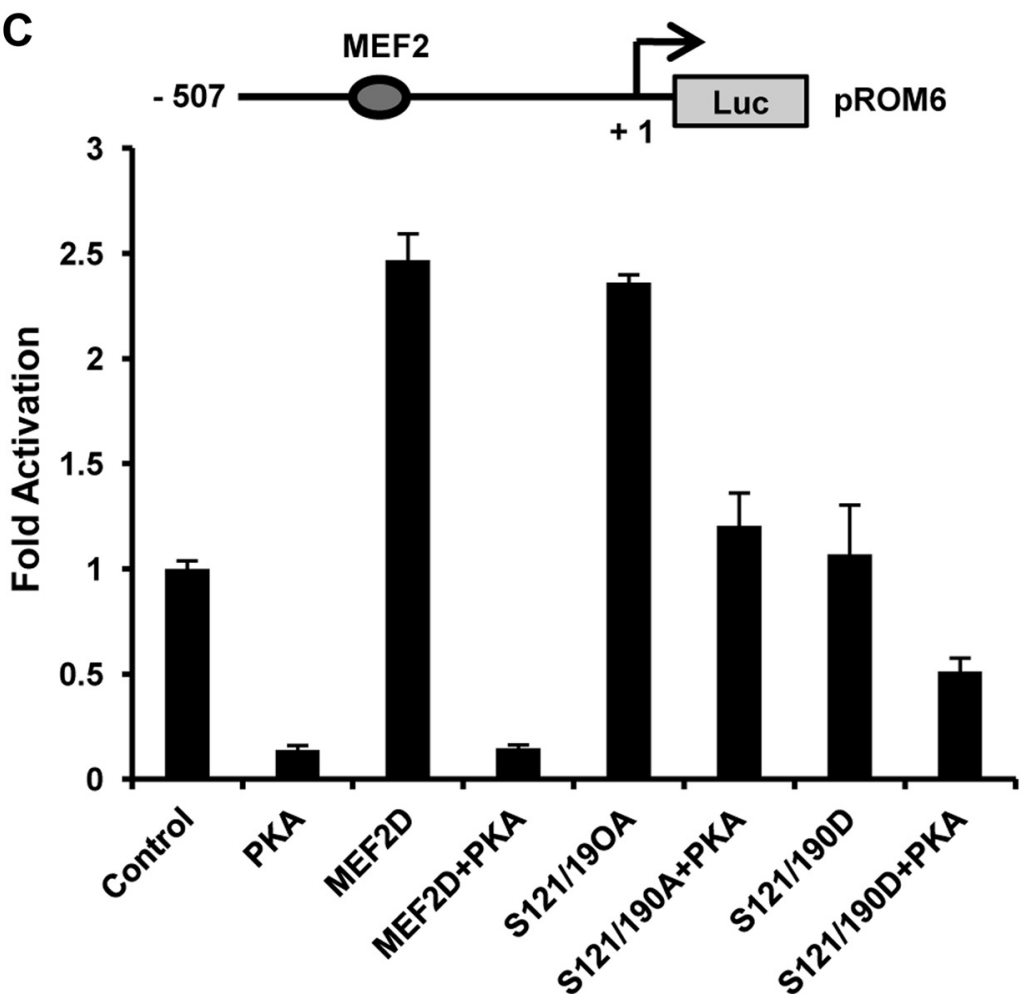

Figure 8. Exogenous KLF6 expression promotes hippocampal neuronal survival. $A$, Primary hippocampal neurons were transfected with expression vectors encoding wtMEF2D, wtKLF6, or empty vector with or without the catalytic subunit of PKA (pFCPKA). Thirty-six hours after transfection, neurons were stained by annexin V-FITC and PI using annexin V-FITC apoptosis detection kit, Sigma. Neuronal apoptosis was determined by flow cytometry analysis (FACS analyzer). B , Detection of KLF6 protein expression. Primary hippocampal neurons were transiently transfected with empty vector or wtKLF6 expression vector. Cell extracts were
PKA-mediated MEF2 repression by HDAC recruitment

Previously, we have documented that HDACs (4 and 5) bind MEF2 with high affinity and, importantly, the mechanism by which PKA inhibits MEF2 transcriptional activity is due to two effects. One is a direct conformational change in the MEF2D protein caused by phosphorylation at Ser 121/190. The other is an indirect mechanism by enhancing the nuclear content of HDACs, which results in an increase in abundance of MEF2/HDAC repressor complexes (Du et al., 2008). The contribution of the latter mechanism varies depending on the opposing signals for nuclear HDAC content. In differentiating skeletal muscle there is a strong stimulus for HDAC nuclear extrusion that counteracts the nuclear retention effects of PKA, whereas in other cell types we reasoned that PKA-mediated nuclear retention of HDACs might account for more of the repressive effects on MEF2 activity (Du et al., 2008). A recent study has also documented that this mechanism occurs in cardiac myocytes (Backs et al., 2011). Interestingly, it has been documented previously that PKA signaling enhances HDAC localization to the nucleus in hippocampal neuronal cells (Belfield et al., 2006). We therefore assessed the contribution of HDAC nuclear retention to the inhibition of MEF2 activity and KLF6 transcription. To begin to elucidate the possible functional role of HDAC4, the endogenous protein level of HDAC4 was documented in primary hippocampal neurons (Fig. 9A). Next, we examined whether PKA signaling mediated any modulation of the cellular localization of endogenous HDAC4 protein. Primary hippocampal neuronal cells were treated with FSK followed by immunofluorescence labeling using polyclonal HDAC4 antibody. As shown in Figure 9B, HDAC4 accumulated in the nucleus in response to FSK compared with that in the solvent treated cells. We further confirmed that PKA signaling promotes the nuclear localization of HDAC4 in hippocampal neu-

\footnotetext{
$\leftarrow$

prepared for immunoblotting analysis as indicated. KLF6 polyclonal antibody (1:1000) was used to detect the KLF6 protein level. Actin (polyclonal, 1:1000) was used as a loading control. C, Cos7 cells were cotransfected with pGL3-KLF6-Luc reporter gene and wtMEF2D and MEF2D double mutants (S121/190A or S121/190D) with or without pFC-PKA. pCMV- $\beta$-galactosidase was used to normalize transfection efficiencies. MEF2mediated transcriptional activity was determined by luciferase and $\beta$-gal assays as described in Material and Methods. (Data are the mean $\pm \mathrm{SEM} ; n=3$.)
} 
A

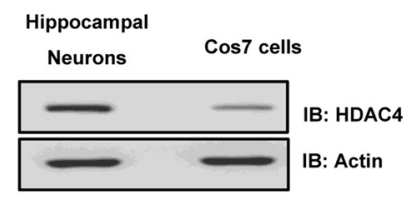

B
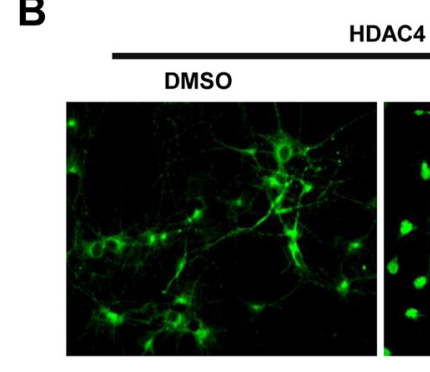

C

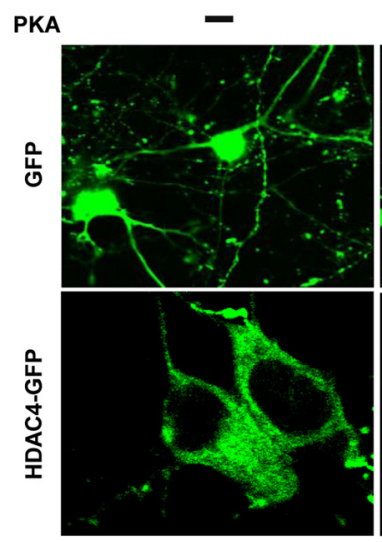

D

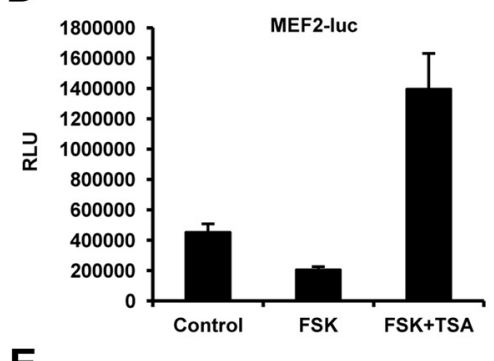

E

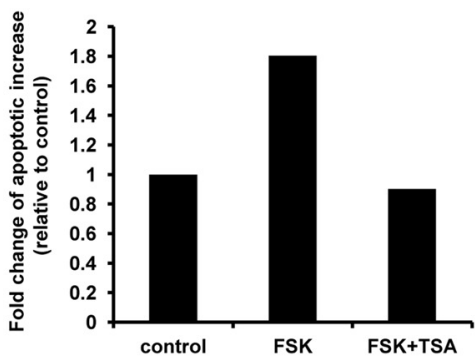

$\mathbf{F}$
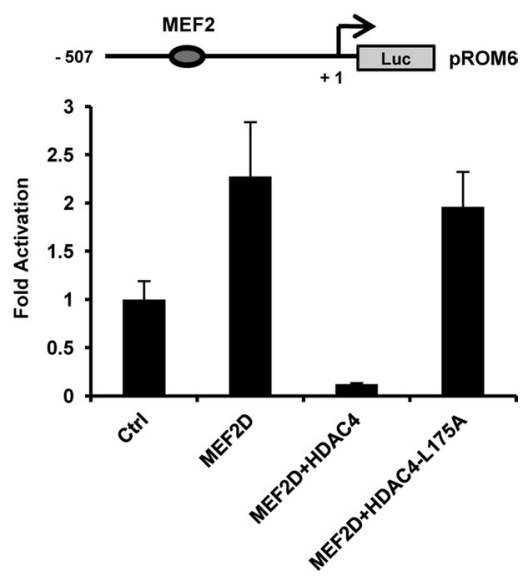

Figure 9. Increased nuclear localization of HDAC4 by PKA signaling in hippocampal neurons. $A$, Cell lysates of primary hippocampal neuronal and Cos7 cells were prepared and equal amounts of total protein were separated by 10\% SDS-PAGE followed by immunoblot analysis. HDAC4 polyclonal antibody (1:1000) was used to detect the endogenous HDAC4 protein. Actin (polyclonal, 1:2000) was used as a loading control. B, Primary hippocampal neuronal cells were treated with $10 \mu \mathrm{m}$ FSK or solvent for $6 \mathrm{~h}$ followed by immunofluorescence labeling using polyclonal HDAC4 antibody. C, Primary hippocampal neurons were cotransfected with GFP alone and GFP-HDAC4 fusion protein, with or without pFC-PKA. Hippocampal neurons expressing GFP alone showed a nonspecific distribution throughout the neuronal cell body including axon, dendrites, and dendritic spines, and this was unaffected by PKA. The shuttling of GFP-HDAC4 from cytoplasm to nucleus in response to PKA was visualized by fluorescence microscopy. D, MEF2 reporter activity is repressed by PKA signaling and rescued by HDACs inhibitor TSA. Primary hippocampal neurons were transiently transfected with pGL3- 4XMEF2-Luc reporter gene and cells were treated with $10 \mu \mathrm{M}$ FSK alone and in combination with $1 \mu \mathrm{M} \mathrm{TSA}$ (as indicated). Thirty-six hours after transfection, cell extracts were prepared for luciferase and $\beta$-gal assays. E, Primary hippocampal neurons were stimulated with $10 \mu \mathrm{m}$ FSK alone and in combination with $1 \mu \mathrm{m}$ TSA for $6 \mathrm{~h}$. Percentage of apoptotic cells was determined by annexin V-FITC apoptosis detection kit, using FACS analyzer. Bar graph represents the changes in number of apoptotic cells. $\boldsymbol{F}$, Primary hippocampal neurons were transiently transfected with pGL3-KLF6-Luc reporter construct and wtMEF2D expression vector alone or in combination with HDAC4 and HDAC4-L175A vectors (as indicated). MEF2-mediated transcriptional activity was determined by luciferase and $\beta$-gal assays as described in Material and Methods.

rons by using a GFP-HDAC4 fusion protein or GFP alone, which was cotransfected with or without activated PKA (Fig. 9C). The GFP-HDAC4 localization data indicates that PKA increases the nuclear levels of GFP-HDAC4 (Fig. 9C, bottom panels) while having no effect on GFP alone (Fig. 9C, top panels). These results are consistent with previous observations, suggesting that PKA signaling promotes the formation of HDAC4/MEF2 repressor complexes by increasing the levels of HDAC4 in the nuclear compartment leading to downstream repression of key MEF2 target genes involved in survival such as KLF6.

To further assess the extent of the HDAC4 contribution to PKA-mediated MEF2 repression, we performed transcriptional reporter gene assays in conjunction with HDAC inhibition using trichostatin A (TSA). Primary hippocampal neurons were trans- fected with a MEF2 reporter gene and cells were treated with FSK alone and in combination with TSA. HDAC inhibition with TSA reduced the FSK effect and partially superactivates MEF2 transcriptional activity (Fig. 9D). Subsequent FACS analysis indicated that treatment with TSA also rescues neuronal cells from apoptosis (Fig. 9E). HDAC4 strongly represses MEF2-dependent transcriptional activation of the KLF6 promoter, and a mutation of HDAC4 (L175A) that has previously been shown not to interact with MEF2, has no effect (Fig. 9F). The lack of interaction between HDAC4-L175A and MEF2D was confirmed in coprecipitation assays (Fig. 10A). Also, the enhanced amount of HDAC4 coprecipitating with MEF2D when active PKA is cotransfected confirms our previous observations. To gain further insight into the role of HDAC4 in neuronal survival, we used siRNA to downregulate HDAC4 expression. First, we tested the 
efficacy of HDAC4 silencing by Western blot analysis. Primary hippocampal neurons (7DIV) were transfected with three independent HDAC4 siRNAs and a control scrambled RNA (scRNA). As shown in Fig. $10 B$, the reduction of HDAC4 protein level was observed in cells expressing siHDAC4 in contrast to cells expressing the scRNA. To examine whether HDAC4 silencing can induce hippocampal neuronal survival, primary hippocampal neurons were transfected with HDAC4siRNA or scRNA. Neuronal apoptosis was quantified by flow cytometry. Depletion of HDAC4 protects neurons from apoptosis when neurons are treated with forskolin (Fig. 10C). These data indicate that the HDAC4-MEF2 interaction is an important component of PKA-mediated MEF2 repression in hippocampal neurons.

\section{KLF6 protects hippocampal neurons from apoptosis}

There is growing evidence that the KLF factors are involved in cell survival and we postulated that the activation of KLF6 could be an important downstream component of the MEF2D-dependent prosurvival pathway. As shown earlier, KLF6 over-expressing cells showed a substantially reduced percentage of apoptotic cells in the presence of PKA compared with PKA alone. To further address the role of KLF6 expression in hippocampal neuronal survival, we used a loss of function assay using siRNA to downregulate KLF6 expression. First, we assessed the efficacy of KLF6 silencing by Western blot analysis. Primary hippocampal neurons (7DIV) were transfected with three independent KLF6 siRNAs and a control scrambled siRNA (scRNA). As shown in Fig. $11 A$, the reduction of KLF6 protein level was observed in cells expressing siKLF6 in contrast to cells expressing the scrambled siRNA. To further examine whether KLF6 silencing can induce hippocampal neuronal apoptosis, primary hippocampal neurons were transfected with KLF6-siRNA or scRNA. Neuronal apoptosis was quantified by flow cytometry as described previously. Depletion of KLF6 enhanced neuronal apoptosis relative to the scRNA (Fig. $11 \mathrm{~B}$ ), suggesting that KLF6 functions as a prosurvival molecule in neuronal cells, although this effect was not as penetrant as MEF2 inhibition. Together, the prosurvival effect of exogenous overexpression KLF6 shown earlier and the modest but evident effects of KLF6 knockdown on survival reveal that KLF6 may be an intrinsic component of the neuronal survival pathway mediated by MEF2.
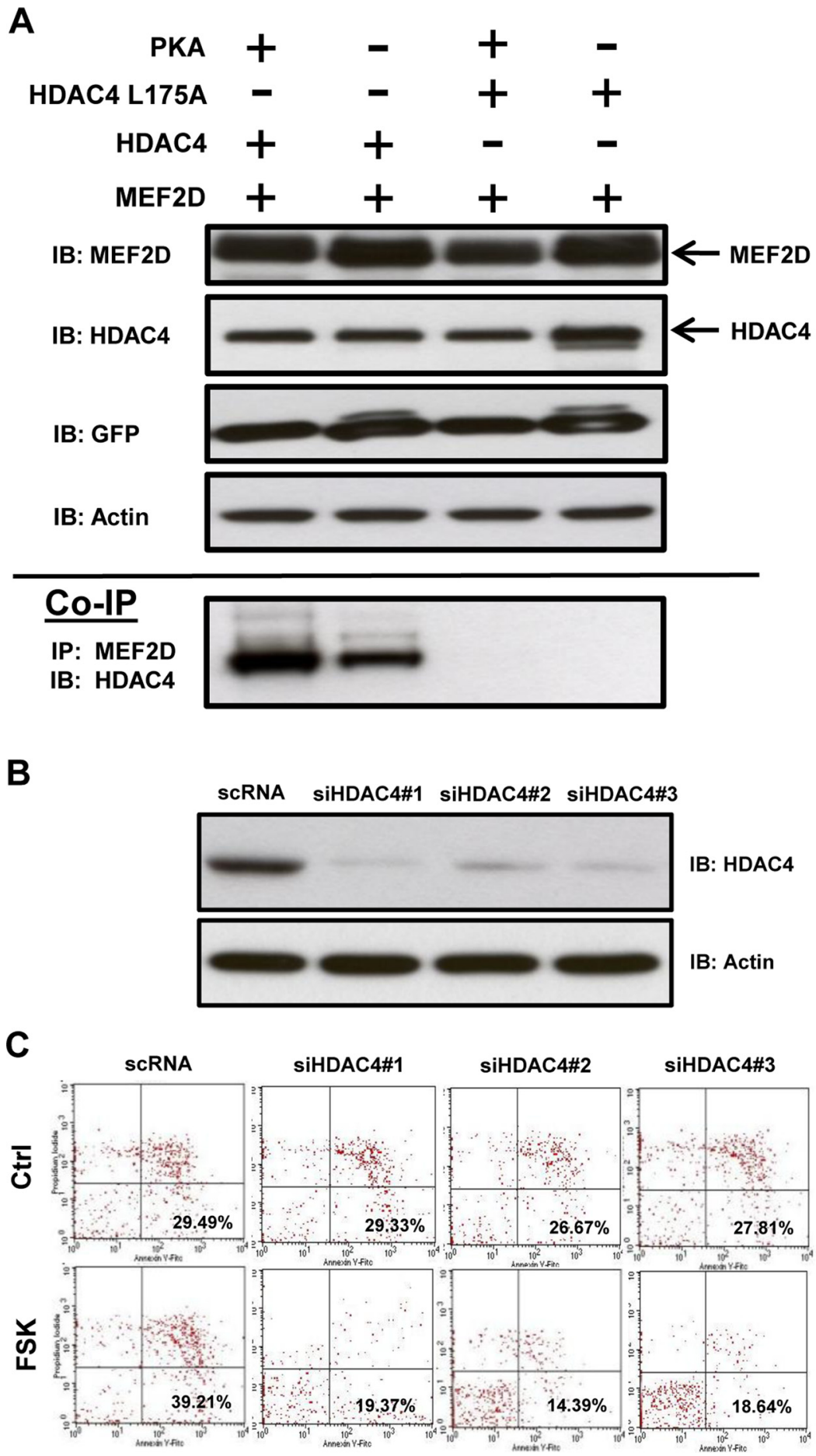

Figure 10. PKA induces physical association of HDAC4 with MEF2D in hippocampal neurons. $\boldsymbol{A}$, Primary hippocampal neurons were transiently transfected with indicated expression plasmids. Cell extracts were immunoprecipitated with anti-MEF2D followed by immunoblotting with anti-HDAC4. Whole-cell lysates were immunoblots with indicated antibodies. HDAC4 interacts with MEF2D in the presence of PKA and this association is lost with HDAC4L175A with or without PKA. $\boldsymbol{B}$, For HDAC4 gene silencing in primary hippocampal neurons, three independent siRNAs were transfected along with a control scRNA. Cells were harvested and lysates were prepared $48 \mathrm{~h}$ later. Protein level of HDAC4 was analyzed by immunoblot using HDAC4 polyclonal antibody (1:1000). Actin (polyclonal, 1:2000) was used as a loading control. C, Primary hippocampal neurons were transfected with two independent siRNAs and a control scRNA. $48 \mathrm{~h}$ after transfection, cells were stained with annexin V-FITC and PI using annexin V-FITC apoptosis detection kit, as described in Material and Methods. Neuronal apoptosis was measured using flow cytometry (FACS analyzer).

\section{Discussion}

Apoptosis has been implicated in the pathogenesis of various neurodegenerative diseases including Alzheimer's and Parkin- 
A

scRNA si-KLF6\#1 si-KLF6\#2 si-KLF6\#3

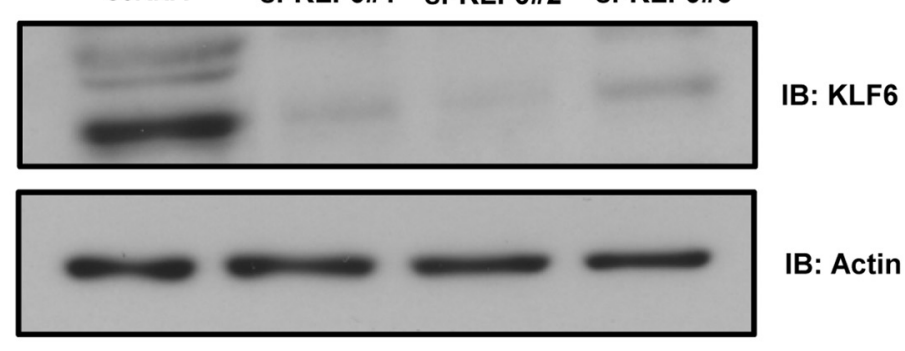

B

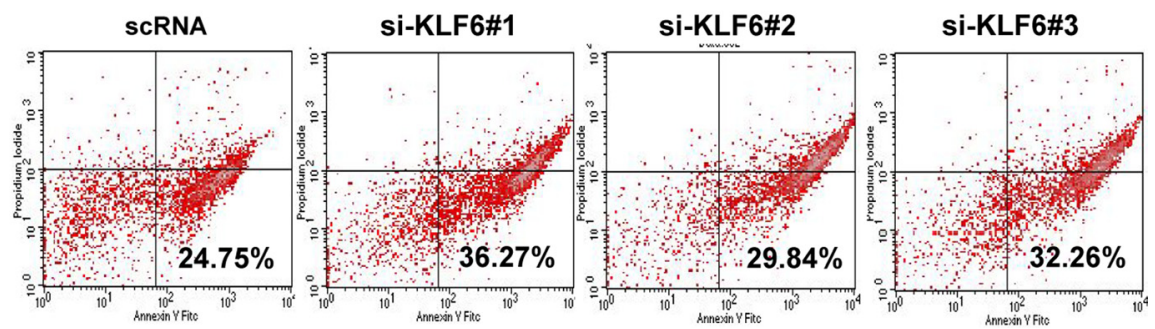

Figure 11. Silencing of KLF6 expression enhances apoptosis in primary hippocampal neurons. A, For KLF6 gene silencing in primary hippocampal neurons, three independent siRNAs were transfected along with a control scRNA. Cells were harvested and lysates were prepared $48 \mathrm{~h}$ later. Equal amounts of total protein were separated by 10\% SDS-PAGE followed by immunoblot analysis using KLF6 polyclonal antibody (1:1000) to detect the KLF6 protein level. Actin (polyclonal, 1:2000) was used as a loading control. $\boldsymbol{B}$, Primary hippocampal neurons were transfected with three independent KLF6 siRNAs and a control scRNA. Forty-eight hours after transfection, cells were stained with annexin V-FITC and PI using annexin V-FITC apoptosis detection kit, as described in Material and Methods. Neuronal apoptosis was measured using flow cytometry.

son's disease (Bredesen, 1995; Gupta et al., 2006; Zhao et al., 2008). The cAMP-PKA signaling pathway regulates a variety of cellular functions and numerous important biological processes. More specifically, PKA signaling has been reported to be associated with the progression of Alzheimer's disease in the hippocampus (Martínez et al., 1999; Lim et al., 2005; Zhao et al., 2008). Modulation of gene expression networks underlying these pivotal cellular events ultimately leading to neurodegeneration is still in its infancy (Crews and Masliah, 2010). Furthermore, neurodegenerative diseases often exhibit alterations in many cellular processes, such as increased oxidative and toxic stress, mitochondrial dysfunction, failure of synaptic activities, and aberrant phosphorylation (Zhang et al., 2008). In this report, we demonstrate that a variety of perturbations of protein kinase $\mathrm{A}$ signaling induce apoptosis of primary hippocampal neurons through inactivation of the prosurvival role of the MEF2D transcription factor. Also, identification of KLF6 as a key downstream component of the MEF2D survival pathway that is downregulated by PKA signaling provides further insight into the physiology and pathophysiology of the nervous system.

Interestingly, a recent report has implicated KLF6 induction in the response to pilocarpine-induced seizures in the hippocampus (Jeong et al., 2011). In view of our data implicating MEF2D as a critical regulator of KLF6 expression, it will be of considerable interest to determine whether MEF2 activity is indeed induced in this pilocarpine-induced seizure model that mimics features of temporal lobe epilepsy (TLE) in humans. Since MEF2 had been implicated as a prosurvival molecule in the CNS, it is tempting to speculate that its induction may well occur in the physiologic response to the pronounced neuronal damage that results from excitotoxic stress. Also, in a model of zebrafish neural regeneration after optic nerve injury, KLF6 knockdown ablates regeneration due to the attenuated expression of KLF6 target gene, Tuba 1a (Veldman et al., 2010). KLF6 expression has been documented in multiple other adult brain regions apart from the hippocampus, including the olfactory bulb, cerebral cortex, amygdale, thalamus, and hypothalamus (Jeong et al., 2009). Moreover, loss of heterozygosity on chromosome 10p in glioblastoma (Camacho-Vanegas et al., 2007) has implicated KLF6 as a potential tumor suppressor in this region, and KLF6 expression has also been reported to be suppressed by transcriptional silencing in esophageal squamous cell carcinoma (Yamashita et al., 2002). The requirement for MEF2 for appropriate expression of KLF6 in all of these brain regions and potentially also in cancer models remains to be fully elucidated. In conjunction with the studies reported here, a variety of experimental systems suggest a potential role of the MEF2-KLF6 pathway in neuronal survival and regeneration; future studies to further test this are therefore warranted.

As alluded to above, MEF2 activity has been implicated in a variety of contexts when neuronal survival is challenged and, based on available evidence, it is reasonable to assume that MEF2 plays a role in the orchestration of survival. Conversely, it is perhaps worthwhile to consider what happens to MEF2 activity when an insult is sufficiently severe as to overwhelm the survival response to cause cell death. One study has indeed reported that 4-phenyl-1, 2, 3, 6-tetrahydropyridine (MPTP) induction of neuronal cell death is correlated with cdk5-mediated phosphorylation of MEF2D (Smith et al., 2006), and a further study has documented that 6-hydroxydopamine (6-OHDA) mediated apoptosis in PC12 cells resulted in a marked reduction of MEF2D levels, an effect which was antagonized by treatment with roscovitine, a cdk5 inhibitor (Kim et al., 2011). As well as these studies, our studies presented here show that cAMP-mediated PKA activation can lead to a potent repression of MEF2 activity, which also results in enhanced neuronal apoptosis. Our previous studies (Du et al., 2008), along with these reported here, document a bipartite mechanism of MEF2D regulation by PKA through direct phosphorylation of S121/190 and enhanced assembly of a MEF2D/HDAC4 repressor complex. Strikingly, in view of these other studies that show a mechanistic link between MEF2D and neuronal survival, a recent study has documented a downregulation of MEF2D in nigral neurons in Parkinson's disease patients when compared with those of age-matched controls (Chu et al., 2011). The implication of prosurvival MEF2 activity in a variety of neuronal damage models does suggest the possibility that MEF2 protects against a variety of cellular stresses and toxic insults. We have observed that overexpression of MEF2D does protect hippocampal neurons somewhat from $\mathrm{H} 2 \mathrm{O} 2$ mediated cell death supporting this idea further (data not shown). Further delineation of the complex signaling pathways converging on MEF2 will be important in potentially identifying other cellular signaling pathways that modulate neuronal survival.

There are some studies clearly implicating cAMP in neuronal protection as well as in cell death, and further clarification of this 
issue is warranted. It is possible that the duration and intensity of the signaling could be a factor in determining the cellular outcome. The effects of PKA/cAMP signaling on cell survival also likely depend on cell type, for example, cAMP has been implicated in a neuroprotective role in cerebellar granule neurons (Wang et al., 2005). Conversely, our studies using a variety of perturbations of cAMP signaling (Forskolin, dbcAMP, exogenous expression of the active catalytic subunit of PKA) all indicate the same effect of promoting cell death in cultured primary hippocampal neurons. However, survival of neurons depends on a complex series of cellular and intracellular communication networks mediated by cross talk between signal transduction cascades. Therefore, it is entirely possible that modulation of the type of signaling (e.g., short term versus chronic, high intensity versus low) may shift the balance resulting in a different cellular outcome.

In addition to the well defined survival role of MEF2 in neurons, MEF2 has also been implicated in activity-dependent synapse elimination that fulfills a crucial role in the refinement of neuronal circuitry by altering synapse caliber and number (Flavell et al., 2006; Wierenga et al., 2006; Chandrasekaran et al., 2007; Turrigiano, 2008; Barbosa et al., 2008). Moreover, a recent study has reported that this MEF2-dependent excitatory synapse elimination is lost in hippocampal neurons from mice that are nullyzygous for an RNA binding protein (FMRP) whose function is lost in Fragile $\mathrm{X}$ syndrome, the most prevalent form of human autism and mental retardation (Pfeiffer et al., 2010). Last, and of relevance for our study, it was reported that cocaine administration inhibits striatal MEF2 activity through a cAMP-dependent mechanism (Pulipparacharuvil et al., 2008), and cocaine-mediated increases in dendritic spine density were dependent on MEF2 suppression. Consistent with this idea that MEF2 regulates dendritic morphogenesis, Shalizi et al. (2007) reported that PIASx, which functions as a MEF2 SUMO E3 ligase, represses MEF2 activity in neurons to orchestrate morphogenesis of postsynaptic dendrites. Thus, the regulation of MEF2 activity by PKA may also impinge on MEF2-dependent synapse modulation.

Clearly, the regulation of MEF2 activity and its downstream targets in the CNS by cAMP-PKA signaling requires consideration in terms of understanding the capability to dynamically alter synaptic connectivity and neural circuitry as well as neuronal survival during development, physiology, and pathology of the mammalian CNS.

\section{References}

Backs J, Worst BC, Lehmann LH, Patrick DM, Jebessa Z, Kreusser MM, Sun Q, Chen L, Heft C, Katus HA, Olson EN (2011) Selective repression of MEF2 activity by PKA-dependent proteolysis of HDAC4. J Cell Biol 195:403-415.

Barbosa AC, Kim MS, Ertunc M, Adachi M, Nelson ED, McAnally J, Richardson JA, Kavalali ET, Monteggia LM, Bassel-Duby R, Olson EN (2008) MEF2C, a transcription factor that facilitates learning and memory by negative regulation of synapse numbers and function. Proc Natl Acad Sci U S A 105:9391-9396.

Belfield JL, Whittaker C, Cader MZ, Chawla S (2006) Differential effects of $\mathrm{Ca}^{2+}$ and cAMP on transcription mediated by MEF2D and cAMPresponse element-binding protein in hippocampal neurons. J Biol Chem 281:27724-27732.

Black BL, Olson EN (1998) Transcription control of muscle development by myocyte enhancer factor-2 (MEF2) proteins. Annu Rev Cell Dev Biol 14:167-196.

Bolger TA, Zhao X, Cohen TJ, Tsai CC, Yao TP (2007) The neurodegenerative disease protein ataxin- 1 antagonizes the neuronal survival function of myocyte enhancer factor-2. J Biol Chem 282:29186-29192.

Bredesen DE (1995) Neuronal apoptosis. Ann Neurol 38:839-851.

Butts BD, Linseman DA, Le SS, Laessig TA, Heidenreich KA (2003) Insulin- like growth factor-I suppresses degradation of the pro-survival transcription factor myocyte enhancer factor 2D (MEF2D) during neuronal apoptosis. Horm Metab Res 35:763-770.

Camacho-Vanegas O, Narla G, Teixeira MS, DiFeo A, Misra A, Singh G, Chan AM, Friedman SL, Feuerstein BG, Martignetti JA (2007) Functional inactivation of the KLF6 tumor suppressor gene by loss of heterozygosity and increased alternative splicing in glioblastoma. Int J Cancer 121:1390-1395.

Chandrasekaran AR, Shah RD, Crair MC (2007) Developmental homeostasis of mouse retinocollicular synapses. J Neurosci 27:1746-1755.

Chu Y, Mickiewicz AL, Kordower JH (2011) $\alpha$-Synuclein aggregation reduces nigral myocyte enhancer factor-2D in idiopathic and experimental Parkinson's disease. Neurobiol Dis 41:71-82.

Contestabile A (2002) Cerebellar granule cells as a model to study mechanisms of neuronal apoptosis or survival in vivo and in vitro. Cerebellum 1:41-55.

Cox DM, Quinn ZA, McDermott JC (2000) Cell signaling and the regulation of muscle-specific gene expression by myocyte enhancer-binding factor 2. Exerc Sport Sci Rev 28:33-38.

Crews L, Masliah E (2010) Molecular mechanisms of neurodegeneration in Alzheimer's disease. Hum Mol Genet 19:R12-R20.

Du M, Perry RL, Nowacki NB, Gordon JW, Salma J, Zhao J, Aziz A, Chan J, Siu KW, McDermott JC (2008) Protein Kinase A repress skeletal myogenesis by targeting myocyte enhancer factor 2D. Mol Cell Biol 28:2952-2970.

Eroglu C, Barres BA (2010) Regulation of synaptic connectivity by glia. Nature 468:223-231

Fischer EA, Verpont MC, Garret-Sinha LA, Ronco PM, Rossert JA (2001) KLF6 is a zinc finger protein expressed in a cell specific manner during kidney development. J Am Soc Nephrol 12:726-735.

Flavell SW, Cowan CW, Kim TK, Greer PL, Lin Y, Paradis S, Griffith EC, Hu LS, Chen C, Greenberg ME (2006) Activity-dependent regulation of MEF2 transcription factors suppresses excitatory synapse number. Science 311:1008-1012.

Flavell SW, Kim TK, Gray JM, Harmin DA, Hemberg M, Hong EJ, MarkenscoffPapadimitriou E, Bear DM, Greenberg ME (2008) Genome-wide analysis of MEF2 transcriptional program reveals synaptic target genes and neuronal activity-dependent polyadenylation site selection. Neuron 60:1022-1038.

Gaudilliere B, Shi Y, Bonni A (2002) RNA interference reveals a requirement for myocyte enhancer factor $2 \mathrm{~A}$ in activity dependent neuronal survival. J Biol Chem 277:46442-46446.

Gehrau RC, D'Astolfo DS, Prieto C, Bocco JL, Koritschoner NP (2005) Genomic organization and functional analysis of the gene encoding the Krüppel-like transcription factor KLF6. Biochim Biophys Acta 1730:137-146.

Gordon JW, Pagiatakis C, Salma J, Du M, Andreucci JJ, Zhao J, Hou G, Perry RL, Dan Q, Courtman D, Bendeck MP, McDermott JC (2009) Protein kinase A regulated assembly of a MEF2\{middle dot\}HDAC4 repressor complex controls c-Jun expression in vascular smooth muscle cells. J Biol Chem 284:19027-19042.

Gupta VB, Hegde ML, Rao KS (2006) Role of protein conformational dynamics and DNA integrity in relevance to neuronal cell death in neurodegeneration. Curr Alzheimer Res 3:297-309.

Heidenreich KA, Linseman DA (2004) Myocyte enhancer factor-2 transcription factors in neuronal differentiation and survival. Mol Neurobiol 29:155-166.

Ikeshima H, Imai S, Shimoda K, Hata J, Takano T (1995) Expression of a MADS box gene, MEF2D, in neurons of the mouse central nervous system: implication of its binary function in myogenic and neurogenic cell lineages. Neurosci Lett 200:117-120.

Jeong KH, Kim SK, Kim SY, Cho KO (2009) Immunohistochemical localization of Krüppel-like factor 6 in the mouse forebrain. Neurosci Lett 453:16-20.

Jeong KH, Lee KE, Kim SY, Cho KO (2011) Upregulation of Krüppel-like factor 6 (KLF6) in the mouse hippocampus after pilocarpine-induced status epilepticus. Neuroscience 186:170-178.

Kano M, Hashimoto K (2009) Synapse elimination in the central nervous system. Curr Opin Neurobiol 19:154-161.

Kim MK, Kim SC, Kang JI, Hyun JH, Boo HJ, Eun SY, Park DB, Yoo ES, Kang HK, Kang JH (2011) 6-Hydroxydopamine-Induced PC12 Cell Death is Mediated by MEF2D Down-regulation. Neurochem Res 36:223-231.

Legradi A, Varszegi S, Szigeti C, Gulya K (2011) Adult rat hippocampal 
slices as in vitro models for neurodegeneration: Studies on cell viability and apoptotic processes. Brain Res Bull 84:39-44.

Leifer D, Krainc D, Yu YT, McDermott J, Breitbart RE, Heng J, Neve RL, Kosofsky B, Nadal-Ginard B, Lipton SA (1993) MEF2C, a MADS/ MEF2-family transcription factor expressed in a laminar distribution in cerebral cortex. Proc Natl Acad Sci U S A 90:1546-1550.

Leifer D, Golden J, Kowall NW (1994) Myocyte-specific enhancer binding factor $2 \mathrm{C}$ expression in human brain development. Neuroscience 63:1067-1079.

Li M, Linseman DA, Allen MP, Meintzer MK, Wang X, Laessig T, Wierman ME, Heidenreich KA (2001) Myocyte enhancer factor 2A and 2D undergo phosphorylation and caspase-mediated degradation during apoptosis of rat cerebellar granule neurons. J Neurosci 21:6544-6552.

Lim G, Wang S, Lim JA, Mao J (2005) Activity of adenylyl cyclase and protein kinase A contributes to morphine-induced spinal apoptosis. Neurosci Lett 389:104-108.

Lin X, Shah S, Bulleit RF (1996) The expression of MEF2 genes is implicated in CNS neuronal differentiation. Brain Res Mol Brain Res 42:307-316.

Linseman DA, Cornejo BJ, Le SS, Meintzer MK, Laessig TA, Bouchard RJ, Heidenreich KA (2003) A myocyte enhancer factor 2D (MEF2D) kinase activated during neuronal apoptosis is a novel target inhibited by lithium. J Neurochem 85:1488-1499.

Lømo J, Blomhoff HK, Beiske K, Stokke T, Smeland EB (1995) TGF-beta 1 and cyclic AMP promote apoptosis in resting human B lymphocytes. J Immunol 154:1634-1643.

Lyons GE, Micales BK, Schwarz J, Martin JF, Olson EN (1995) Expression of mef2 genes in the mouse central nervous system suggests a role in neuronal maturation. J Neurosci 15:5727-5738.

Martínez M, Fernández E, Frank A, Guaza C, de la Fuente M, Hernanz A (1999) Increased cerebrospinal fluid cAMP levels in Alzheimer's disease. Brain Res 846:265-267.

McDermott JC, Cardoso MC, Yu YT, Andres V, Leifer D, Krainc D, Lipton SA, Nadal-Ginard B (1993) hMEF2C genes encodes skeletal muscle and brain specific transcription factors. Mol Cell Biol 13:2564-2577.

McKinsey TA, Zhang CL, Olson EN (2002) MEF2: a calcium-dependent regulator of cell division, differentiation and death. Trends Biochem Sci $27: 40-47$

Myklebust JH, Josefsen D, Blomhoff HK, Levy FO, Naderi S, Reed JC, Smeland EB (1999) Activation of the cAMP signalling pathway increases apoptosis in human B-precursor cells and is associated with downregulation of Mcl-1 expression. J Cell Physiol 180:71-80.

Naya FJ, Olson E (1999) MEF2: a transcriptional target for signaling pathways controlling skeletal muscle growth and differentiation. Curr Opin Cell Biol 11:683-688.

Ornatsky OI, McDermott JC (1996) MEF2 protein expression, DNA binding specificity and complex composition, and transcriptional activity in muscle and non-muscle cells. J Biol Chem 271:24927-24933.

Perry RL, Yang C, Soora N, Salma J, Marback M, Naghibi L, Ilyas H, Chan J, Gordon JW, McDermott JC (2009) Direct interaction between myocyte enhancer factor 2 (MEF2) and protein phosphatase lalpha represses MEF2-dependent gene expression. Mol Cell Biol 29:3355-3366.

Pfeiffer BE, Zang T, Wilkerson JR, Taniguchi M, Maksimova MA, Smith LN, Cowan CW, Huber KM (2010) Fragile X mental retardation protein is required for synapse elimination by the activity-dependent transcription factor MEF2. Neuron 66:191-197.

Potthoff MJ, Olson EN (2007) MEF2: a central regulator of diverse developmental programs. Development 134:4131-4140.

Pulipparacharuvil S, Renthal W, Hale CF, Taniguchi M, Xiao G, Kumar A, Russo SJ, Sikder D, Dewey CM, Davis MM, Greengard P, Nairn AC, Nestler EJ, Cowan CW (2008) Cocaine regulates MEF2 to control synaptic and behavioral plasticity. Neuron 59:621-633.

Sands WA, Palmer TM (2008) Regulating gene transcription in response to cyclic AMP elevation. Cell Signal 20:460-466.

Schulz RA, Chromey C, Lu MF, Zhao B, Olson EN (1996) Expression of the D-MEF2 transcription in the Drosophila brain suggests a role in neuronal cell differentiation. Oncogene 12:1827-1831.

Shalizi A, Gaudillière B, Yuan Z, Stegmüller J, Shirogane T, Ge Q, Tan Y, Schulman B, Harper JW, Bonni A (2006) A calcium-regulated MEF2 sumoylation switch controls postsynaptic differentiation. Science 311:1012-1017.

Shalizi A, Bilimoria PM, Stegmüller J, Gaudillière B, Yang Y, Shuai K, Bonni A (2007) PIASx is a MEF2 SUMO E3 ligase that promotes postsynaptic dendritic morphogenesis. J Neurosci 27:10037-10046.

Smith PD, Mount MP, Shree R, Callaghan S, Slack RS, Anisman H, Vincent I, Wang X, Mao Z, Park DS (2006) Calpain-regulated p35/cdk5 plays a central role in dopaminergic neuron death through modulation of the transcription factor myocyte enhancer factor 2. J Neurosci 26:440-447.

Turrigiano GG (2008) The self-tuning neuron: synaptic scaling of excitatory synapses. Cell 135:422-435.

Veldman MB, Bemben MA, Goldman D (2010) Tubala gene expression is regulated by KLF6/7 and is necessary for CNS development and regeneration in zebrafish. Mol Cell Neurosci 43:370-383.

Wang X, Tang X, Li M, Marshall J, Mao Z (2005) Regulation of neuroprotective activity of myocyte enhancer factor-2 by cAMP-protein kinase A signaling pathway in neuronal survival. J Biol Chem 280:16705-16713.

Wierenga CJ, Walsh MF, Turrigiano GG (2006) Temporal regulation of the expression locus of homeostatic plasticity. J Neurophysiol 96:2127-2133.

Yamashita K, Upadhyay S, Osada M, Hoque MO, Xiao Y, Mori M, Sato F, Meltzer SJ, Sidransky D (2002) Pharmacologic unmasking of epigenetically silenced tumor suppressor genes in esophageal squamous cell carcinoma. Cancer Cell 2:485-495.

Yang CC, Ornatsky OI, McDermott JC, Cruz TF, Prody CA (1998) Interaction of myocyte enhancer factor 2 (MEF2) with a mitogen-activated protein kinase, ERK5/BMK1. Nucleic Acids Res 26:4771-4777.

Zhang L, Zambon AC, Vranizan K, Pothula K, Conklin BR, Insel PA (2008) Gene expression signatures of cAMP/protein kinase A (PKA)-promoted, mitochondrial-dependent apoptosis. Comparative analysis of wild-type and cAMP-deathless $\$ 49$ lymphoma cells. J Biol Chem 283:4304-4313.

Zhao L, Qian ZM, Zhang C, Wing HY, Du F, Ya K (2008) Amyloid betapeptide 31-35-induced neuronal apoptosis is mediated by caspasedependent pathways via cAMP-dependent protein kinase A activation. Aging Cell 7:47-57.

Zhu B, Ramachandran B, Gulick T (2005) Alternative pre-mRNA splicing governs expression of a conserved acidic transactivation domain in myocyte enhancer factor 2 factors of striated muscle and brain. J Biol Chem 280:28749-28760. 